\title{
Initial mass function effects on the colour evolution of disk galaxies ${ }^{\star}$
}

\author{
P. Westera, M. Samland, S. J. Kautsch, R. Buser, and K. Ammon
}

\begin{abstract}
Astronomical Institute. Department of Physics and Astronomy, University of Basel, Venusstr. 7, 4102 Binningen, Switzerland e-mail: [westera; buser] aastro.unibas.ch
\end{abstract}

Received 17 July 2006 / Accepted 21 December 2006

\section{ABSTRACT}

\begin{abstract}
Aims. In this work, we want to find out if the IMF can be determined from colour images, integrated colours, or mass-to-light ratios, especially at high redshift, where galaxies cannot be resolved into individual stars, which would enable us to investigate dependencies of the IMF on cosmological epoch.

Methods. We use chemo-dynamical models to investigate the influence of the Initial Mass Function (IMF) on the evolution of a Milky Way-type disk galaxy, in particular of its colours.

Results. We find that the effect of the IMF on the internal gas absorption is larger than its effect on the light from the stellar content. However, the two effects work in the opposite sense: An IMF with more high mass stars leads to brighter and bluer star-light, but also to more interstellar dust and thus to more absorption, causing a kind of "IMF degeneracy". The most likely wavelength region in which to detect IMF effects is the infrared (i.e., $J H K$ ). We also provide photometric absorption and inclination corrections in the SDSS ugriz and the HST WFPC2 and NICMOS systems.
\end{abstract}

Key words. stars: luminosity function, mass function - galaxies: evolution - galaxies: ISM - dust, extinction - galaxies: photometry

\section{Introduction}

The determination of the Initial Mass Function (IMF) of stellar populations and the detection of its possible variations are long lasting questions in astronomy. The first IMF, a singleslope power law, was published by Salpeter (1955) based on stars in the solar neighbourhood, and is still occasionally used in stellar population studies. However, it has been known since Miller \& Scalo's (1979) milestone paper on the subject that the IMF flattens at low masses. In the meantime, this finding has been confirmed in numerous works (Scalo 1986; Kroupa et al. 1993; Gould et al. 1997; Reid \& Gizis 1997; Gould et al. 1998; Chabrier 2001; Piotto \& Zoccali 1999; Zoccali et al. 2000, and others). Recently, Chabrier (2002) also found indications of a turn-over in the brown dwarfs regime. For recent reviews see Kroupa (2002), Chabrier (2003).

An indication of the importance of the subject is the number of IMFs produced over the years: Salpeter (1955), Miller \& Scalo (1979), Lequeux (1979), Kennicutt (1983), Scalo (1986), Ferraro et al. (1997), Piotto et al. (1997), Scalo (1998), Carigi et al. (1999), Kroupa (2001) (universal and present day IMFs), Chabrier (2001), and others.

Another important issue are variations of the IMF with the star forming conditions (pressure, density, metallicity of the forming cloud, etc.). Although such variations are predicted, only little evidence of them has been found so far (Kroupa 2001). We expect especially at high redshift to see differences from the present-day IMF, as the lower metallicity of the star-forming clouds is expected to cause higher temperatures and thereby higher average stellar masses (Larson 1998). Unfortunately, high redshift galaxies are too faint to be resolved into individual stars,

^ Appendices A-D are only available in electronic form at http://www . aanda.org which makes it difficult, if not impossible, to determine their IMFs. Therefore, it would be interesting to know if other, more global observables, such as integrated spectra or colours, can also yield some information about the stellar IMF. The IMF influences the light of a galaxy not only directly through the contributions of the stars of different (birth) masses, but also indirectly by affecting the entire evolution. The fraction of high mass stars determines the gas- and "dust"-yield of the partial populations and, hence, the star formation history ( $\mathrm{SFH}$ ) from the second stellar generation on, as well as the gas absorption. For these reasons, we hope to be able to see signatures of the IMF even in the integrated light of galaxies.

For this purpose, Portinari et al. (2004) studied the influence of the IMF on the ( $I$ band) mass-to-light $(M / L)$ ratio of galactic disks $(\mathrm{Sbc} / \mathrm{Sc})$ using chemo-photometric models and adopting 6 different IMFs, including the Salpeter (1955) and Kroupa (1998) IMFs, which is interesting in connection with this work, because in the present work, we also compare models using the Salpeter IMF and a more recent IMF by Kroupa. For each IMF, they calculate chemical evolution models with infall, metallicity gradients, and SFHs representative of late-type spiral disks (but not varying with the IMF). They find that so-called "bottomlight" IMFs (i.e., with less low-mass stars than Salpeter) yield low $M / L$ ratios $(\sim 0.7-1)$, in agreement with various dynamical arguments and cosmological simulations. However, they calculate only stellar $M / L$ ratios without taking into account gas absorption.

In this work, we use fully consistent 3-dimensional chemodynamical models by M. Samland to calculate the evolution of two galaxies, with the same boundary conditions (cosmology, gas infall history, etc.), but adopting different IMFs: the Salpeter and the Kroupa (2001) "universal" IMFs. Although these are not the most state-of-the-art IMFs available, they were chosen 
because of their clear differences in their low-to-high-mass stars ratios, so any IMF-induced differences should appear clearly. The spectra and (Hubble Space Telescope (HST), SLOAN Digital Sky Survey (SDSS) and Washington) colour images and integrated colours were then calculated using the same method as in Westera et al. (2002b). An important advantage of our programme is that we can disentangle different effects on the spectral properties of a model galaxy, such as of internal absorption, by artificially blinding these contributions out, and then recalculating the spectral properties.

The outline of this paper is as follows: Sect. 2 describes the physics and properties of the two chemo-dynamical models, and in Sect. 3, it is explained how the spectral properties of these models were calculated. Section 5 contains the results, and a comparison of the model colours with SDSS data, which were extracted in the way described in Sect. 4. In Sect. 6, we draw some conclusions and give a brief summary.

\section{The chemo-dynamical models}

The models simulate the formation of a $8 \times 10^{11} M_{\odot}$ galaxy leaving all parameters, except for the IMF, the same for both models. The models are for a Milky Way-type galaxy, but we expect our results (IMF effects) to be similar for other disk galaxies, as the properties, which are important for the colour evolution (star formation history, gas feedback and enrichment, etc.) probably depend in a similar way on the IMF for different types of disk galaxies.

The two models have different IMFs implemented, a Salpeter (1955) IMF, which is a one-segment potential law with an exponent $\alpha=2.35$ in the mass range from 0.1 to $50 M_{\odot}$, and a Kroupa "universal" (also called "standard" or "canonical") IMF (Eq. (2) of Kroupa 2001), a two-segment law with $\alpha=1.3$ from 0.08 to $0.5 M_{\odot}$ and $\alpha=2.3$ from 0.5 to $50 M_{\odot}$, respectively, in order to investigate the influence of the IMF shape on the formation processes of a disk galaxy. The main difference between the two IMFs lies in the high-to-low mass stars ratio, in the sense that a population with a Kroupa IMF has more high-mass stars than a population of the same mass, but with a Salpeter IMF.

The 3-dimensional chemo-dynamical models are of the same type as those described in Samland \& Gerhard (2003); so here, we only summarise very briefly the main properties, but take a more detailed look at the few quantities that will become interesting for the interpretation in Sect. 5: the (stellar) mass surface density, the stellar particle ages, the stellar metallicities, and the gas density and metallicity.

The models take into account initial cosmological and environmental conditions, but also internal feedback processes, such as heating by supernovae, dissipation, radiative cooling, nucleosynthesis, and in- and outflows. Since they are fully selfconsistent models, they include dark matter, stars and the different phases of the interstellar medium (ISM), as well as the processes ("chemistry") which connect the ISM and the stars.

More quantitatively, the models assume a flat Universe with the following cosmological parameters: $H_{0}=70 \mathrm{~km} \mathrm{~s}^{-1} \mathrm{Mpc}^{-1}$, $\Omega_{0}=0.3, \Omega_{\Lambda}=0.7$, and $\frac{M_{\text {Bary }}}{M_{\text {Dark }}}=\frac{1}{8}$. The total spin parameter of the model galaxies was chosen to be $\lambda=0.05$ (Barnes \& Efstathiou 1987), and the angular momentum distribution was calculated according to Bullock et al. (2001) using $\mu=10$. We follow the evolution from $z=9.5$ (corresponding in this cosmology to an age of the Universe of $0.5 \mathrm{Gyr})$ until $z=0(13.5 \mathrm{Gyr})$.

The models are characterised by a slowly growing dark halo and a continuous gas infall following the universal mass

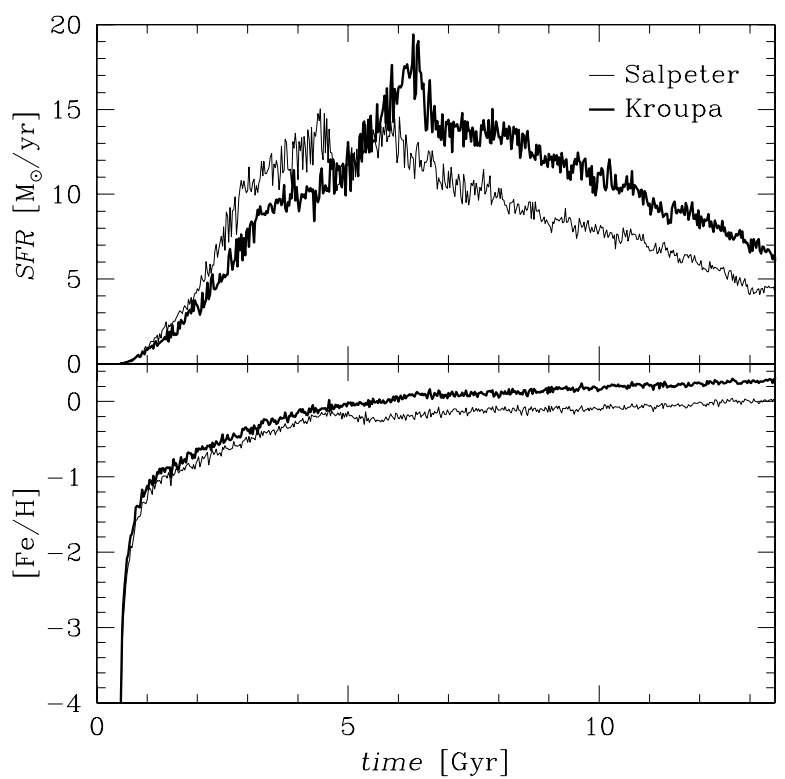

Fig. 1. Star formation histories of the Salpeter (thin) and Kroupa (thick) models. Top panel: star formation rates, bottom panel: average star formation metallicities.

accretion histories found by van den Bosch (2002) and Wechsler et al. (2002) using a formation redshift $z_{\text {Formation }}$ of 4.0 and a total mass $M$ of $8 \times 10^{11} M_{\odot}$. As the gas infall continues until the present day, we expect for both models a mixture of stellar populations of many different ages.

However, the two models do not show the same SFH. As the Kroupa IMF features more high mass stars, star formation will result in a stronger heating by stellar radiation, more stellar wind, more feedback from supernovae I and II and thus a higher mass return and metal yield. As a result, the Kroupa model has a lower SFR than the Salpeter model for the first $\sim 5 \mathrm{Gyr}$, due to the heating from the stellar winds from the first generations. Afterwards, that is after $\sim 5.5 \mathrm{Gyr}$, the Kroupa model has a higher SFR due to the larger available amount of gas (as seen in Fig. 2, top panel). After around 7.5 Gyr, this higher SFR has compensated for the lower SFR in the beginning, so from that point on the total stellar mass is higher in the Kroupa model galaxy. In the end (at 13.5 Gyr or redshift 0 ), the total stellar mass amounts to $1.07 \times 10^{11} M_{\odot}$ in the Salpeter model and to $1.25 \times 10^{11} M_{\odot}$ in the Kroupa model. The two SFRs can be studied in detail in Fig. 1 (top panel), from which can also be seen that the SFR remains significant until the present epoch, as expected from the gas infall history. The bottom panel shows the average star formation metallicity.

The average gas metallicities $[\mathrm{O} / \mathrm{H}]$ of the models (shown in Fig. 2, middle panel) increase most steeply during the phases of maximum star formation. They start at $[\mathrm{O} / \mathrm{H}] \simeq-4$, and reach their present values of $\sim-0.1$ dex or $\sim+0.2$ dex at $z \simeq 1$. This higher gas metallicity of the Kroupa model, combined with the higher gas density (top panel of Fig. 2), causes the Kroupa model galaxy to contain about twice as much metals in the interstellar matter (ISM), or "dust", as the Salpeter galaxy, which can be seen in the bottom panel of Fig. 2.

The output quantities of interest (which are the input quantities for the programme which calculates the spectral properties) are the following, at each time step: a number of stellar particles, each with its spatial position, initial mass, age, and metallicity, as well as the gas density and metallicity on a 3-dimensional grid 


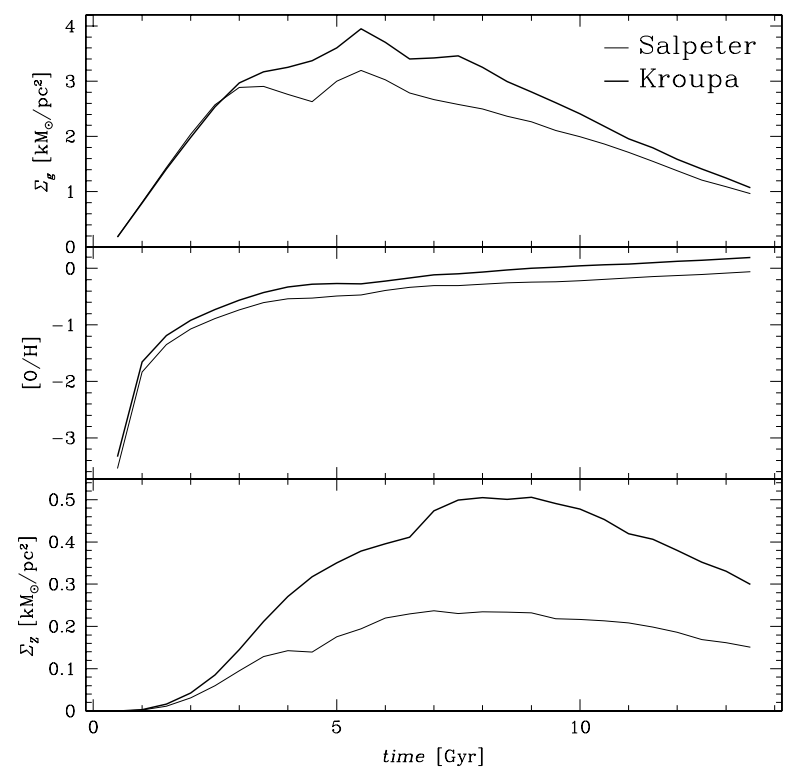

Fig. 2. Average gas column densities (top panel), gas metallicities (middle panel), and "dust" gas column densities, that is $Z_{g} * \Sigma_{g}$, of the Salpeter (thin) and Kroupa (thick) models.

covering the galaxy out to where the gas density is negligible $(100 \mathrm{kpc})$, as a function of time.

Figure 3 shows the stellar mass distributions of the two models projected face-on and edge-on. Both models result in disk galaxies with weak spiral arms, whereas the Salpeter model produces a bulge 2 Gyr sooner (at $\sim 4$ Gyr) than the Kroupa model, which can be seen in the second row of Fig. $3(z=1.2156$, which corresponds to $5 \mathrm{Gyr}$ ). In the Kroupa model (right two panels), the bulge is not yet present at this epoch, but in the Salpeter model (left two panels), it is. This can also be seen in the profiles (Fig. 4, second row). This delay in bulge formation in the Kroupa model is probably also due to stellar winds. As soon as the bulge appears in either model, we also see a plateau in the mass profiles at around 2 to $5 \mathrm{kpc}$. This is due to stellar winds from the bulge, which push out the gas from the inner disk (at $2 \mathrm{kpc}$ ) to a distance of $4 \mathrm{kpc}$, where Star Formation then takes place. It is not bar-induced as in the more massive galaxy studied by Samland \& Gerhard (2003). The fact, that, in Fig. 3, the Kroupa model galaxy seems to have a thicker disk than the Salpeter one, is just a by-eye impression. We calculated the thick and thin disk scaleheights for both models as functions of time, but found no significant differences between the models.

\section{From theoretical quantities to colours and spectra}

To derive 2-dimensional colour images (HST (WFPC2 and NICMOS), SDSS ugriz, Washington CNT 1T2, and other photometric systems) from the star and gas distributions of the galaxy models, we proceeded in the following way:

First, two libraries of simple stellar population (SSP) spectra were produced: one with a Salpeter IMF from 0.1 to $50 M_{\odot}$, and one with a Kroupa IMF from 0.08 to $50 M_{\odot}$, in accordance with the galaxy models. With the Bruzual \& Charlot (2000) Galaxy Isochrone Spectral Synthesis Evolution Library (GISSEL) code (Charlot \& Bruzual 1991; Bruzual \& Charlot 1993, 2003), integrated spectra (ISEDs) of populations were calculated for a grid of population parameters consisting of
7 metallicities $([\mathrm{Fe} / \mathrm{H}]=-2.252,-1.65,-0.65,-0.35,0.09$, 0.447 , and 0.748 ) and $221 \mathrm{SSP}$ ages ranging from 0 to $20 \mathrm{Gyr}$. As input, we used Padova 1994 isochrones (Fagotto et al. 1994; Girardi et al. 1996). There exist more recent versions of the Padova isochrones, Padova 2000 (Girardi et al. 2000), but there is some doubt as to whether these newer tracks produce better agreement with observed galaxy colours than the Padova 1994 models (Bruzual \& Charlot 2003). Furthermore, the Padova 1994 isochrones cover a wider range of metallicities. The spectral library used was the BaSeL 3.1 "WLBC 99" (Westera 2001; Westera et al. 2002a) stellar library. The spectra of this ISED library contain fluxes at 1221 wavelengths from $9.1 \mathrm{~nm}$ to $160 \mu \mathrm{m}$, comfortably covering the entire range where galaxy radiation from stars is significant. The GISSEL software also has a higher resolution stellar library implemented, STELIB, which has a resolution high enough to study spectral (absorption) lines ( $1 \AA$ in the relevant wavelength range), but, with 6900 flux points per spectrum, these spectra proved too large in terms of memory and CPU time to be included in our programme.

After choosing (through three angles) the viewing direction with respect to the galaxy principal plane, and the size (up to $320 \times 320$ pixels) and resolution for the "virtual CCD camera", the stellar particles are grouped into pixels. For each stellar particle, the spectrum is (geometrically, flux point by flux point) interpolated from the ISED library. For metallicities lower than the range covered by the library, the spectra for the lowest metallicity $([\mathrm{Fe} / \mathrm{H}]=-2.252)$ were used. This should not pose any problems, as trends of spectral properties with metallicity are expected to become weak below $[\mathrm{Fe} / \mathrm{H}]=-2.0$, and these lowestmetallicity stellar particles become negligible in number very soon. For SSPs of $50 \mathrm{Myr}$ and younger, we added nebular emission to the spectra in the same way as described in Leitherer et al. (1999), and accordingly removed the flux below $912 \AA$. On the other hand, the emission of HII regions is not implemented. The inclusion of HII regions, as well as planetary nebulae and supernovae, will be one of the next steps in improving the programme.

Then, the spectra were reddened as follows, using the gas density and metallicity in the model to trace the threedimensional distribution of dust: For each stellar particle, the metallicity-weighted gas density was integrated along the line of sight to derive the absorption coefficient $A_{V}$ according to Quillen \& Yukita (2001):

$A_{V}=\frac{1}{50 \frac{M_{\odot}}{\mathrm{pc}^{2}}} \int_{\mathrm{LOS}} \rho_{g}(r)\left(\frac{Z(r)}{Z_{\odot}}\right) \mathrm{d} r$.

The spectrum of the stellar particle was then reddened using the extinction law of Fluks et al. (1994).

All the spectra of stellar particles from the same pixel were added up to give the integrated absolute spectrum of the pixel, which was then redshifted and dimmed using the redshift $z$ from the models, and calculating the distance modulus $m-M$ according to Carroll et al. (1992):

$$
\begin{aligned}
m-M(z)= & 5 \log \left(\frac { c } { H _ { 0 } } ( 1 + z ) \int _ { 0 } ^ { z } \left[\left(1+z^{\prime}\right)^{2}\left(1+\Omega_{M} z^{\prime}\right)\right.\right. \\
& \left.\left.-z^{\prime}\left(2+z^{\prime}\right) \Omega_{\Lambda}\right]^{-1 / 2} \mathrm{~d} z^{\prime}\right)+25
\end{aligned}
$$

We then corrected the spectra for Lyman line blanketing and Lyman continuum absorption by absorption systems at cosmological distances using the formulae given by Madau (1995) for QSO absorption systems. Finally, apparent HST (WFPC2 


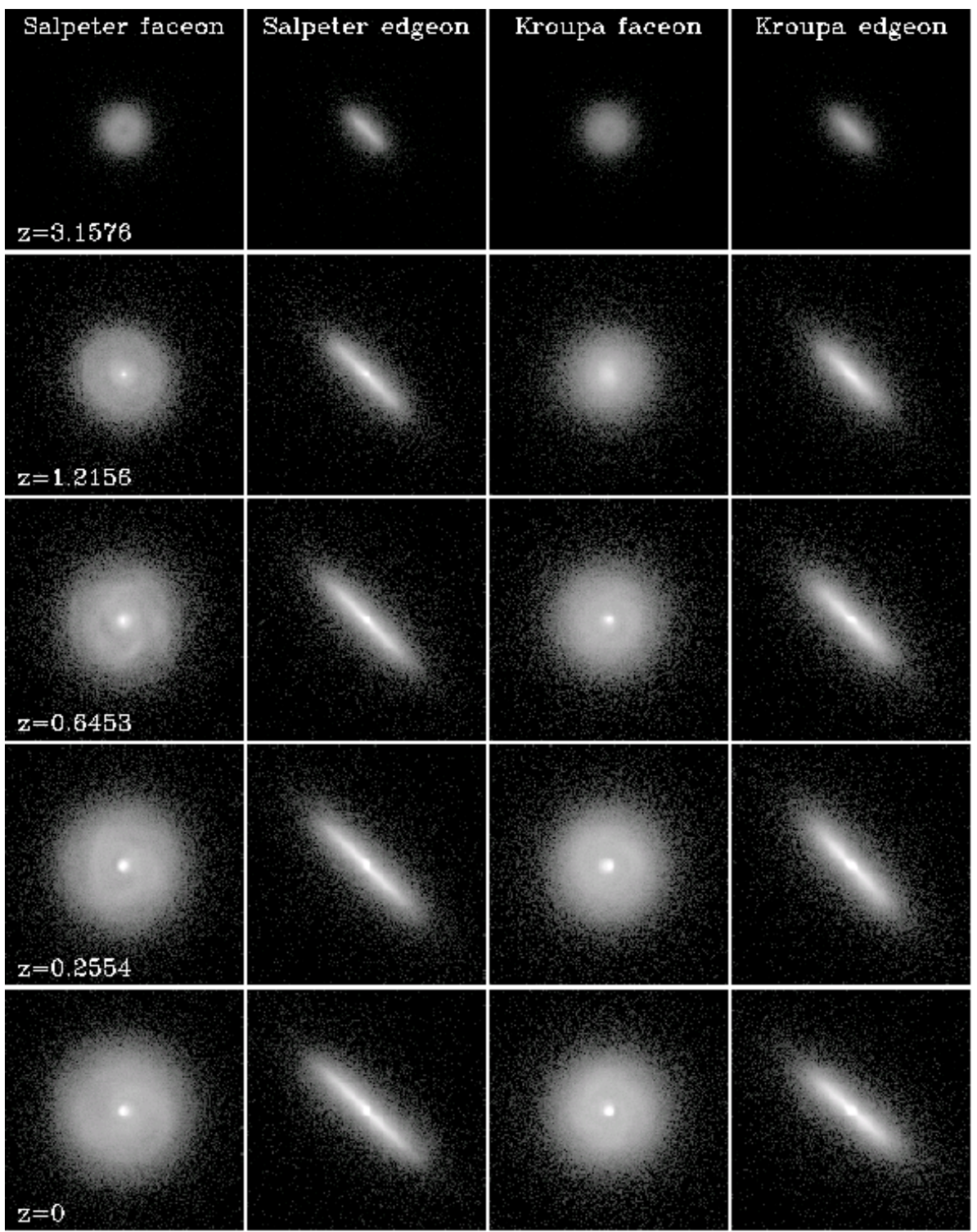

Fig. 3. Stellar mass distributions of the Salpeter and Kroupa models at different redshifts/ages (the redshifts correspond to ages of 2, 5, 7.5, 10.5 , and $13.5 \mathrm{Gyr}$ ) seen face-on and edge-on. The colour scale is logarithmic and covers four orders of magnitudes. The images show an area of $40 \times 40 \mathrm{kpc}$. and NICMOS), SDSS ugriz, Washington CNT1T2 colours and magnitudes were calculated for each pixel through synthetic photometry. Other photometric systems, i.e. Johnson-Cousins UBVRIJHKLM, Strömgren $u b v y$, Kron RI, are also implemented in the programme, but we limited our study to the above-mentioned systems for memory - and CPU time reasons. Including more systems is unlikely to yield further discoveries, since the filter bands of those systems lie in the same wavelength range as the ones of the systems we used, and will thus most probably show the same behaviour with IMF (and other) variations. Furthermore, the HST and SDSS systems seemed most likely to allow extensive comparison with observational data.

At the same time, the absolute (rest frame) spectra and the apparent spectra of all the pixels were added up to derive the absolute and apparent integrated spectra of the galaxy. Examples of such integrated (intrinsic, that is unredshifted and all with the same distance modulus) spectra are shown in Fig. 5. The metallicity-dependent distribution of the stars and the spatially resolved treatment of the gas absorption are the most important for the spectra and colours.

On these integrated spectra, synthetic photometry was performed, too. For computer memory reasons, the spectra of individual pixels or stellar particles were not stored, so the final output quantities of the programme are:

1. a 2-dimensional colour image of the model galaxy, including the effect of internal absorption in intrinsic magnitudes of up to $320 \times 320$ pixels, as seen from a freely chosen angle,

2 . the same image in apparent (redshifted and corrected for the distance modulus and Lyman line blanketing) magnitudes,

3 . the integrated intrinsic spectrum of the entire galaxy plus integrated intrinsic colours and absolute magnitudes,

4. the integrated apparent spectrum of the entire galaxy plus integrated redshifted colours and apparent magnitudes.

Our programme also includes the possibility to account for Galactic foreground reddening. But since this option only makes sense for specific applications, where the foreground reddening is known, it was not used in this work.

These quantities were calculated for both the Salpeter IMF and the Kroupa IMF models, at ages from $0.5 \mathrm{Gyr}$ (corresponding to $z=9.5$, or $0.3 \mathrm{Gyr}$ after the beginning of the simulation) to $13.5 \mathrm{Gyr}$ (the present day) in steps of $0.5 \mathrm{Gyr}$, and from three different directions: face-on, inclined by $60^{\circ}$, henceforth called the diagonal view, and edge-on. The size of a pixel was chosen to be $0.25 \mathrm{kpc}$. Higher resolution would make no sense, as the 

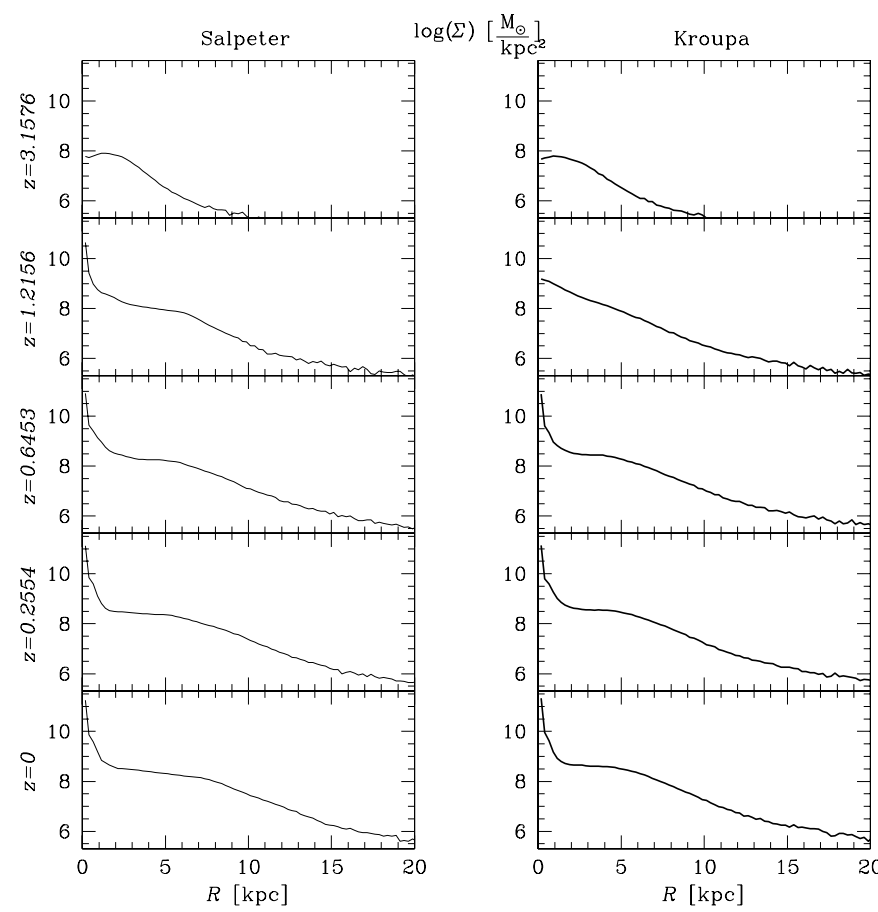

Fig. 4. Stellar (face-on) mass profiles of the Salpeter and Kroupa models at different redshifts/ages. The profiles correspond to the first and third columns of Fig. 3.

galaxy model has a precision of only $0.37 \mathrm{kpc}$. The entire "camera" was chosen $320 \times 320$ pixels wide, thus representing a field of view of $80 \times 80 \mathrm{kpc}$.

To identify absorption effects, the same photometric properties were calculated for both models without internal absorption. Thus, the differences between the regular models and these ones should reflect absorption effects, or the error in models that do not include internal absorption. These models will be called the absorptionless models, and will be used in Sect. 5 .

\section{Data extraction}

In order to test our models, we compare them to galaxy data from the Sloan Digital Sky Survey (SDSS) (York et al. 2000), which offers a large sample of galaxies. The current volume of the SDSS is the Data Release 4 (DR4) which covers in the imaging mode about 180 million unique objects in an area of 6670 square degrees and 849920 spectra within 4783 square degrees (Adelman-McCarthy et al. 2005). We use the SDSS Batch Query Services ${ }^{1}$ on the DR4 Galaxy Table View and SpecObj Table View. This web interface allows to perform queries on the available SDSS archives using the Structured Query Language (SQL). The Galaxy Table View contains optical parameters of all galaxies at the time of the data release. The spectral properties of the galaxies are given in the SpecObj Table View. We remove all objects which are flagged with one or more of the PhotoFlags as given in the Galaxy Table View: blended (object had multiple peaks detected within it); edge (object is too close to edge of frame of the survey); saturated (object contains saturated pixels); ellipfaint (not measured isophotal properties and incomplete profiles). These flags allow us to reject all objects near the survey borders and blended with spikes of nearby stars. As our model colours differ for the face-on and edge-on

\footnotetext{
${ }^{1}$ http://casjobs.sdss.org/CasJobs/default.aspx
}

view (see Sect. 5), we extract two samples, one for either viewing direction.

For the edge-on sample, we adopt the query used by Kautsch et al. (2006) to collect a catalog of edge-on galaxies, wherein the axial ratio $a / b$ is chosen to be $>3, a$ and $b$ being the major and minor angular isophotal axes in the $g$ band; the major axis $a$ is chosen larger than 15 pixels (which corresponds to 5.94 arcsec) and colours in the ranges $-0.3<g-r<3 \mathrm{mag}$ and $-0.3<$ $r-i<3 \mathrm{mag}$, in order to exclude spurious objects and other artefacts. We limit the sample to a Petrosian magnitude ${ }^{2}$ in the $g$ band of 20 .

For the face-on sample, $a / b$ is chosen to be smaller than 1.5, the isophotal major axis $a$ is also $>15$ pixels, and the colours again lie in the ranges $-0.3<g-r<3$ mag and $-0.3<r-i<3$ mag. Here the Petrosian $g$ band magnitude is limited to 19 mag, since galaxies seen face-on appear brighter than the same ones seen edge-on (see Sect. 5). Using these limiting magnitudes, the two samples contain a similar number of galaxies.

The following biases affect our selection: "Shredded galaxies," i.e., these galaxies are detected as two or more independent objects (this is found in particular for extended objects with substructure and diameters $\geqslant 1^{\prime}$ ); galaxies with unusual colours caused by an AGN and/or dust. Due to these effects we lose less than $1 \%$ of the targets from the SDSS database as estimated from a by-eye-inspection of randomly selected subsamples.

Wrong classification can be the result of various causes: (i) "inverse shredding", where objects arranged in chains are detected as a single object; (ii) bars or spiral arms in faint disks being classified as edge-on galaxies. However, we estimate that these effects affect about $2 \%$ of the targets only.

\section{Results}

In Fig. 6, we see the calculated intrinsic urz band images of the Salpeter and Kroupa model galaxies, both in the same magnitude scaling. The images confirm that the bulge forms later in the Kroupa model (at around 6 Gyr) than in the Salpeter model ( 4 Gyr), as mentioned in Sect. 2. Apart from that, the images of the models look extremely similar. In this section, we will explain why.

\subsection{Intrinsic magnitudes and mass-to-light ratios}

In Tables A.1 to A.4 are listed the intrinsic bolometric, $V$ Johnson, SDSS ugriz, and HST (WFPC2 and NICMOS) magnitudes of the two models integrated over the full galaxies in the diagonal view. We calculated the Washington magnitudes as well, and they can also be calculated for other colour systems, such as Johnson-Cousins UBVRIJHKLM, Strömgren $u b v y$, and Kron $R I$. The evolution of the magnitudes show some oscillations around their mean tendencies, which are the result of shadowing of the bulge by dense streams of infalling molecular gas. They only show up in the diagonal view when absorption is included. One should keep in mind that this can be an additional source of scatter when looking at observed magnitudes and colours of galaxies.

2 The Petrosian magnitudes are derived from the Petrosian flux using a circular aperture centered on every object. The advantage of this method is that this allows an unbiased measurement of a constant fraction of the total galaxy light using the technique based on that of Petrosian (1976). For a detailed description of the Petrosian parameters used in the SDSS we refer to Blanton et al. (2001) and Yasuda et al. (2001). 


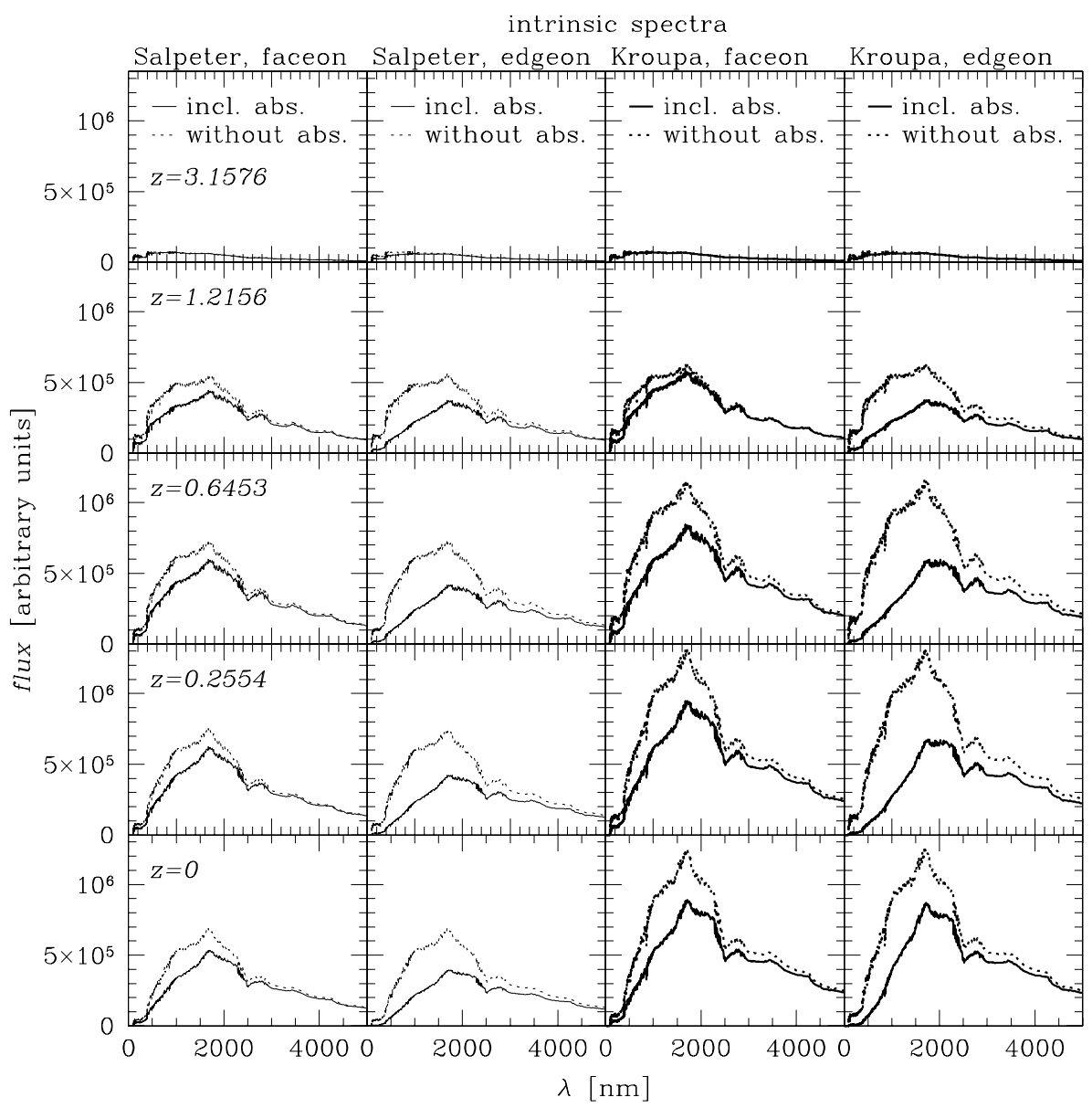

Fig. 5. Intrinsic spectra of the Salpeter (left two columns) - and Kroupa (right two columns) models at five different epochs (different rows), both face-on (Cols. 1 and 3) and edge-on (Cols. 2 and 4), and both without (dotted) and including (solid) absorption.
In the following, most figures concerning magnitudes will show the SDSS $u$ band and the HST NICMOS K222 band, and most figures concerning colours will show $u-g$ and $J 110-$ $K 222$, which are the bluest and reddest calculated magnitudes and colours, respectively, and therefore are the most illustrative of the range of possible effects on colours and magnitudes. For other passbands and colours, the trends will usually lie between the trends of the ones shown in the figures.

Tables B. 1 to B. 4 give the stellar mass-to-light ratios $(M / L)$ of the two models both including and omitting absorption, in bolometric light, Johnson $\mathrm{Vj}$, the SDSS ugriz system, and the HST NICMOS $J H K$ system. They are shown in Fig. 7 in $u, r$, $i$, and $K 222$ as a function of time since the Big Bang. We see that, from around $6 \mathrm{Gyr}$ on, the Kroupa model has $M / L$ s about one third lower than the Salpeter model. It is $\sim 0.5$ mag brighter, due to its higher SFR (see Fig. 1). Unfortunately, when absorption is included, this effect is partly canceled out by the higher gas content of the Kroupa model to a level varying between 0 $(K 222)$ and $0.4 \mathrm{mag}(u)$, which is probably undetectable. The effect in colour bands bluewards of $i$ is, that the two models become indistinguishable, an unfortunate coincidence we call "IMF degeneracy". It is weakest in the $K 222$ band, where the absorption is smaller and the difference between the Salpeter and Kroupa models remains around $\sim 0.5 \mathrm{mag}$, leaving the difference in $M / L$ unaltered, but even this will be difficult to measure.

The above-mentioned absorption effects vary with the viewing angle, as is illustrated for the Kroupa model in Fig. 8 (for the Salpeter model they look similar). It shows the $u-$ and $K 222$ evolution of the model in all angles, as well as the absorptionless model (which has the same intrinsic magnitudes viewed from any angle). The lower panels show the absorption effects on these magnitudes (that is the differences between the models with different viewing angles and their absorptionless counterparts). These differences translate into differences in magnitudes between the different inclinations. They amount to $1.5 \mathrm{mag}$ in $u$, and still $0.5 \mathrm{mag}$ in $K 222$, which is more than the IMF effects. Nevertheless, it will be difficult to say something about the orientation of an unresolved galaxy by its intrinsic magnitude alone, since too many things can affect the total magnitude of a real distant galaxy, besides inclination.

\subsection{Intrinsic colours}

If IMF effects on magnitudes or Mass-to-light ratios are insignificant, suffering from a degeneracy, does the IMF manifest itself more strongly in the colours? In Fig. 9, top panel, we see the time evolution of $(u-g)_{0}$ and $(J 110-K 222)_{0}$ of both the Salpeter (thick) - and the Kroupa (thin) models, including absorption (solid) and without (dashed). The bottom panel shows the differences in these colours between the two models, both including absorption (solid) and without (dashed). The thin dotted line shows zero level.

For the $(u-g)_{0}$ colour, we see the same conspiracy between SFR and absorption as before. The colour difference in unabsorbed starlight between the two models (bottom left panel dashed line), which was already very small from the beginning (below $0.1 \mathrm{mag}$ ), is even diminished by the absorption. In $(J 110-K 222)_{0}$, on the other hand, the differences between the Salpeter - and the Kroupa models increase when absorption is taken into account, but they remain too small to be measured 


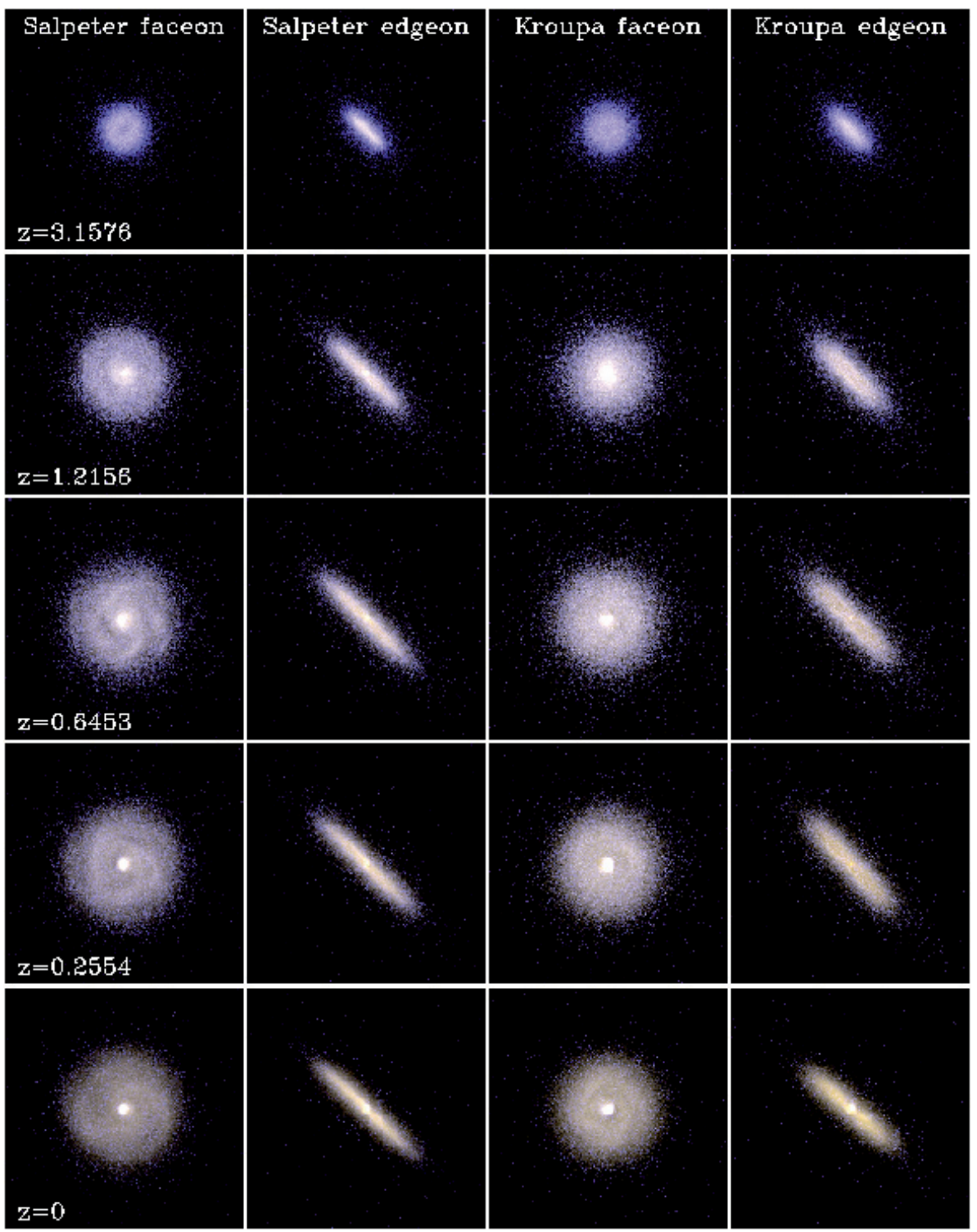

Fig. 6. Calculated intrinsic urz images of the Salpeter and Kroupa models, face-on and edgeon, both in the same magnitude scaling, where the calculated $u$ distribution makes up for the blue portion of the composite image, the $r$ distribution for the yellow portion, and the $z$ distribution for the red portion. The images correspond to the images in Fig. 3. (only up to $0.2 \mathrm{mag}$ ). In colours made up of two magnitudes from widely separated wavelength regions, such as $(u-K 222)_{0}$, they even reach $0.5 \mathrm{mag}$, but in relation to the larger variation of these colours, these differences are less expressive than the 0.2 mag in $(J-K)_{0}$.

In Fig. 10, we see the effect of reddening on the bluest and the reddest colours of our study, $(u-g)_{0}$ and $(J 110-K 222)_{0}$, respectively. The top panels show the time evolution of these two colours for the Kroupa model in all three viewing angles, as well as the absorptionless case, which is the same for all viewing angles. The bottom panel shows the differences between the absorbed and the unabsorbed models in all three inclinations. Clearly, the absorption effects on the bluest colours are small, only up to $\sim 0.2 \mathrm{mag}$ in $(u-g)_{0}$. In $(J 110-K 222)_{0}$, on the other hand, they reach up to $0.8 \mathrm{mag}$ (edge-on), so this colour might be suitable to detect orientation effects. It may seem counterintuitive, that inclination-induced reddening on the bluest colour is much smaller than in the near infrared. This must be because the differential extinction in $J 110$ vs. $K 222$ is much larger than in $u$ vs. $g$.

Having calculated galactic models both omitting dust effects and, as a novelty, self-consistently including them, gives us the unique occasion to test the usual assumption that the combined absorption and reddening effects do not significantly alter colour- $M / L$ relations (Bell \& de Jong 2001). Figure 11 shows four such relations, combining colours and $M / L$ ratios from the reddest and the bluest wavelength regions of our calculations. For the relations involving the $(u-g)_{0}$ colour (left panels), the assumption works reasonably well. The dust effects move the colours and the $M / L$ ratios along the main relations, thereby keeping them in place. For the relations involving $J 110-K 222$ (right panels), on the other hand, the colour- $M / L$ relations are shifted by around $0.2 \mathrm{mag}$ to the red, when absorption is included (The dust affects this colour much more than the $M / L$ ratios). It seems difficult to keep these relations intact by altering the colour and the $M / L$ ratios at the same time, since they are not linear in the first place. A systematic investigation shows, that the assumption can be used for colours involving passbands bluewards of the SDSS $i$ band.

\subsection{Apparent magnitudes}

In practice, apparent magnitudes (redshifted and corrected for distance) are more relevant than intrinsic magnitudes, as they are the quantities that are actually observed. They are given in Tables A.5 to A.8 for the same bands as in Tables A.1 to A.4. 


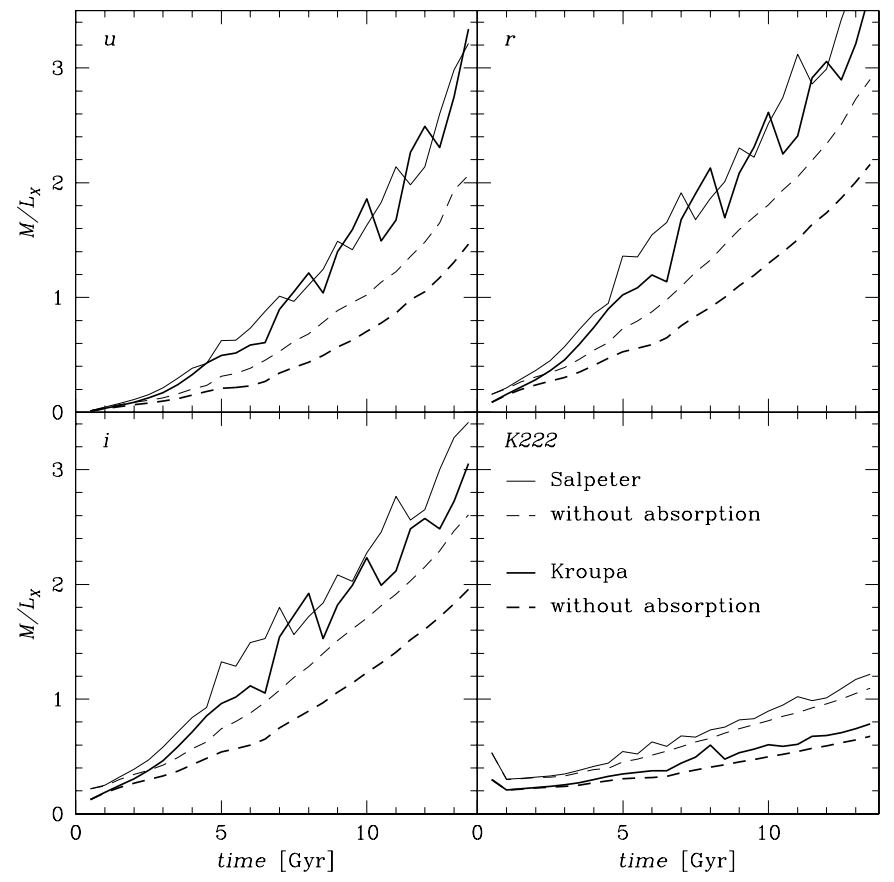

Fig. 7. Stellar mass-to-light ratio evolution in $u, r, i$, and $K 222$ of the Salpeter (thin) and the Kroupa (thick) models, both including (solid) and omitting (dashed) gas absorption.

Due to the very low fluxes at the highest redshift $(9.5116$, corresponding to a Universe age of $0.5 \mathrm{Gyr}$ ), the calculation of the magnitudes at this age suffers too much from precision errors, so these values should be taken with a grain of salt, especially in the bluer passbands. In the bluest bands ( $u, g, U 336$, and $B 439)$, the errors might even affect the second time step (redshift 5.6177) as well, especially when combining the magnitudes to calculate colours, on which small differences have a much more dramatic effect than on magnitudes.

For the $u$ and $K 222$ bands, the redshift evolution is shown in the top panels of Fig. 13, again for both models in the diagonal view, and both including - and not including absorption, like in the first and fourth panels of Fig. 7. In the bottom panels, we see the differences between the Kroupa and the Salpeter models. These differences between the two models show the same tendencies as for the intrinsic magnitudes $(\sim 0.5$ in the absorptionless case, reduced when absorption is included), and are probably even harder to detect than they would be in the intrinsic magnitudes, since they are dominated by distance modulus effects. Again, the $K 222$ band is slightly better for detecting the differences (they remain around 0.5 mag even after including absorption) than the SLOAN filters, but still not good enough.

The absorption effects on the Kroupa model, that is the magnitude differences between the absorptionless and the absorbed model, are given in all calculated passbands in Tables C.1 and C. 2 for the diagonal view. Together with the inclination corrections given in Tables C. 3 to C.6, they can be calculated for all three viewing angles. By subtracting the inclination or absorption corrections for two magnitudes, the inclination or absorption (reddening) corrections for the corresponding colour can be derived. This can be useful to make absorption corrections to galactic models. We only give these corrections for the Kroupa model, because this model is more realistic, and the corrections are very similar for the Salpeter model. As expected, the absorption is the strongest in the edge-on view and the weakest faceon, but the latter does not differ much from the diagonal case.

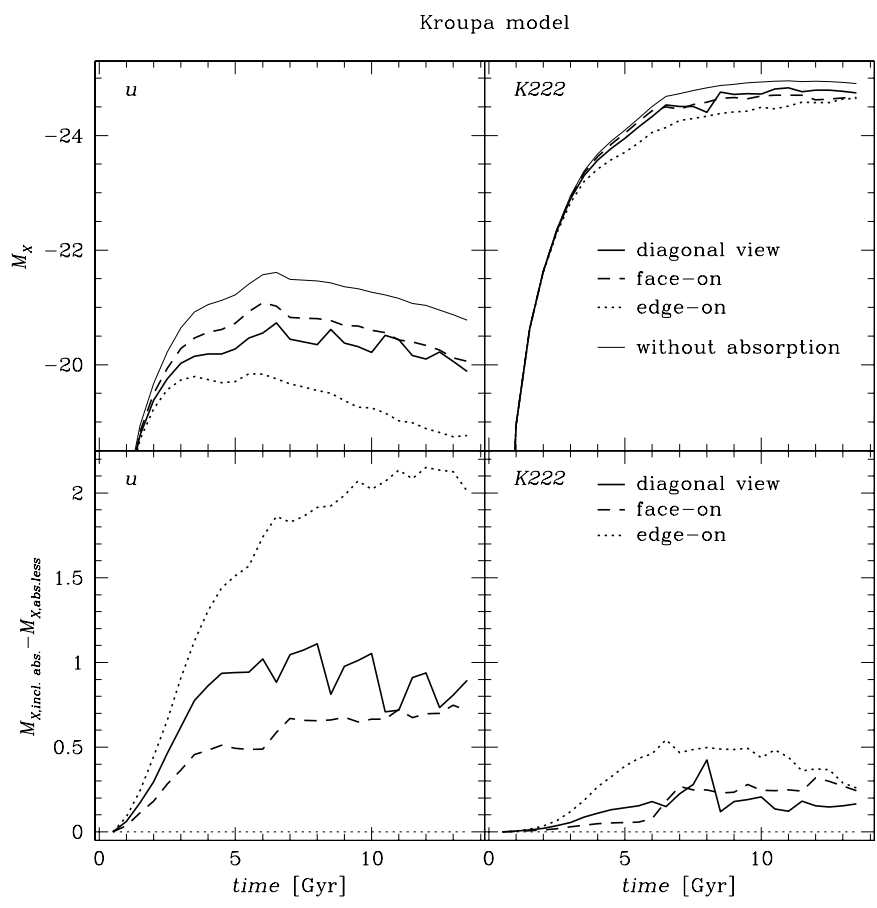

Fig. 8. Top panels: absolute $u$ and $K 222$ magnitude evolution of the Kroupa model as seen in the diagonal view (solid), face-on (dashed), and edge-on (dotted). The thin solid line shows the evolution of the absorptionless model (in the diagonal view, but it looks the same for the face-on and edge-on views). Bottom panels: effects of the gas absorption on the absolute $u$ and $K 222$ magnitudes of the Kroupa models (that is, the differences between the absorptionless and the regular models) as seen in the diagonal view (solid), face-on (dashed), and edge-on (dotted). The thin dotted lines show the zero level.

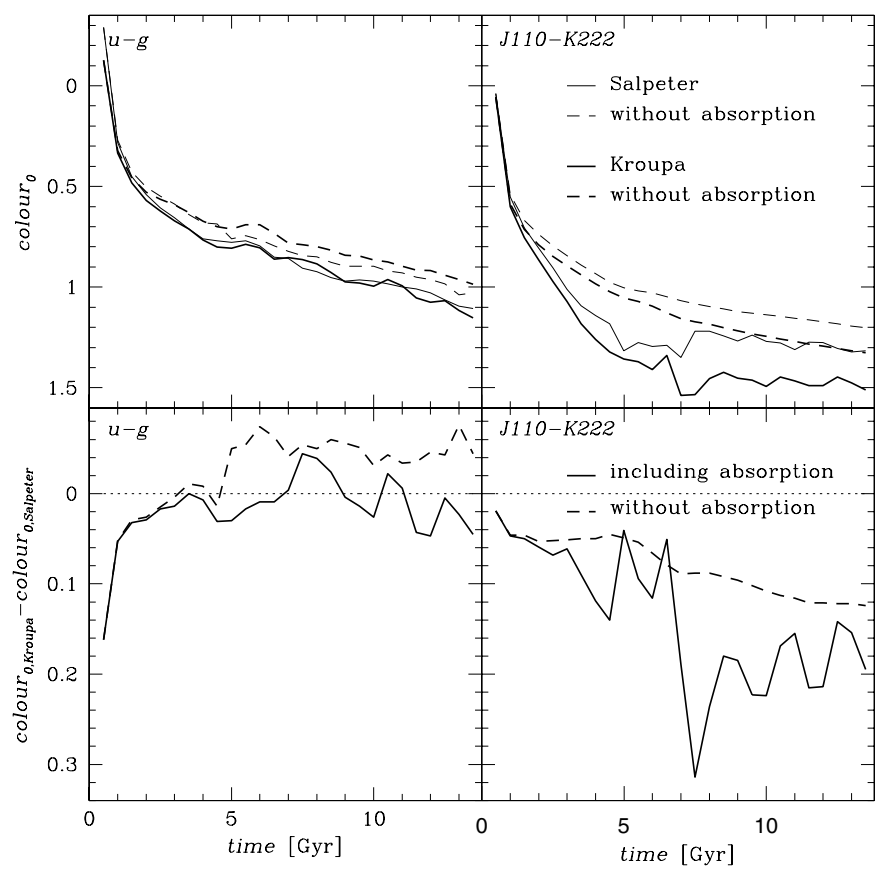

Fig. 9. Top panels: intrinsic $(u-g)_{0}$ and $(J 110-K 222)_{0}$ colour evolution of the Salpeter (thin) and the Kroupa (thick) models, both including (solid) and omitting (dashed) gas absorption. Bottom panel: differences in intrinsic $(u-g)_{0}$ and $(J 110-K 222)_{0}$ colours between the Salpeter and the Kroupa models, both including (solid) and omitting (dashed) gas absorption. The thin dotted line shows the zero level. 


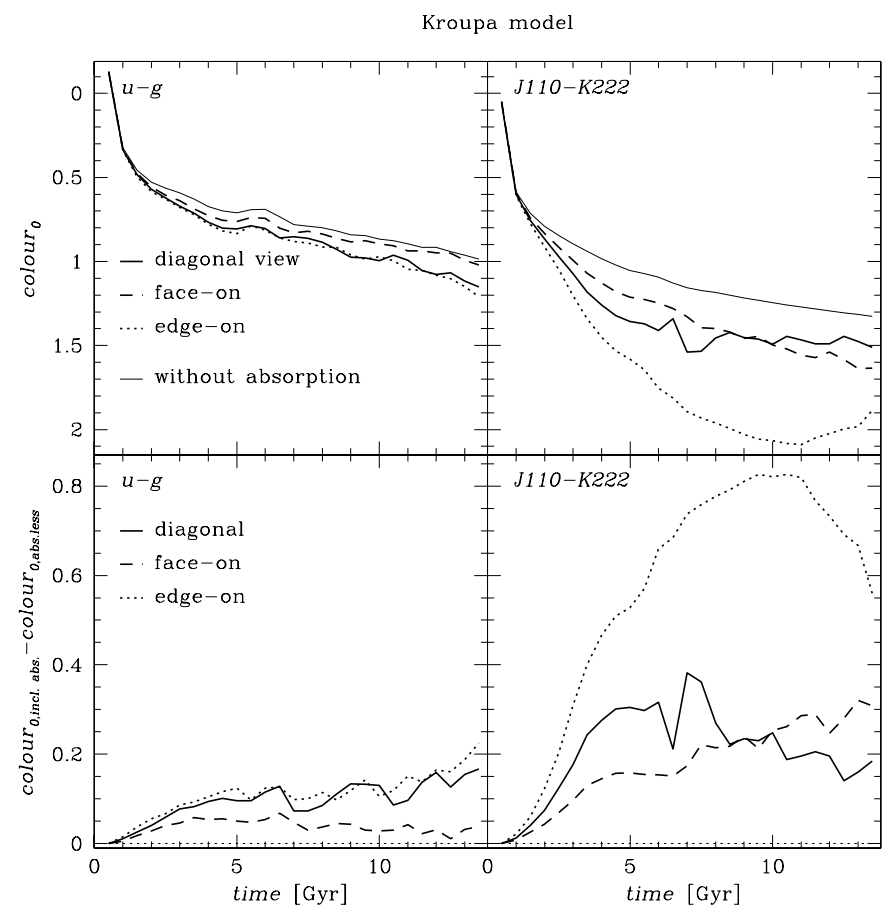

Fig. 10. Top panels: intrinsic $(u-g)_{0}$ and $(J 110-K 222)_{0}$ colour evolution of the Kroupa model as seen in the diagonal view (solid), face-on (dashed), and edge-on (dotted). The thin solid line shows the evolution of the absorptionless model (in the diagonal view, but it looks the same for the face-on and edge-on views). Bottom panels: absorption effects on the intrinsic $(u-g)_{0}$ and $(J 110-K 222)_{0}$ colours of the Kroupa models in each of the three viewing directions, that is, the differences between the absorbed and the absorptionless model seen in the diagonal view (solid), face-on (dashed), and edge-on (dotted). The thin dotted lines show the zero level.

So even for face-on galaxies, it will be impossible to infer the IMF from an integrated magnitude, whereas for edge-on galaxies, the situation is even worse.

If the apparent magnitudes calculated from the models cannot be used to discriminate between the two different IMFs, do they at least reproduce the empirical data? In order to compare our model magnitudes with those from Sect. 4 that represent similar galaxies as in our models, we reduced both data sets (edge-on and face-on) to those galaxies with similar sizes (Petrosian radii) and structure (concentration indices) as the model galaxies. Ideally, one should compare the models to galaxies with the same mass and morphological type, but since these quantities are not given in the SDSS, we resort to size and concentration parameters. More precisely, we determined petroR90 $0_{\mathrm{r}}$ and $\mathrm{C}_{\mathrm{r}}$ as a function of redshift $z$ for both the Salpeter and the Kroupa models, face-on and edge-on, and then reduced the data sets to those galaxies that fulfilled the following criterion:

$0.5 \cdot \operatorname{petroR} 90_{\mathrm{r}, \text { mod }}(z)<$ petroR90 $0_{\mathrm{r}, \mathrm{emp}}(z)$

$<2.0 \cdot \operatorname{petroR} 90_{\mathrm{r}, \mathrm{mod}}(z) \wedge$

$0.98 \cdot \mathrm{C}_{\mathrm{r}, \bmod }(z)<\mathrm{C}_{\mathrm{r}, \mathrm{emp}}(z)<1.02 \cdot \mathrm{C}_{\mathrm{r}, \bmod }(z)$

where petroR90 $90_{r, \bmod }(z)$ and $\mathrm{C}_{\mathrm{r}, \bmod }(z)$ are the Petrosian radius and concentration index calculated from the models and petroR $90_{\mathrm{r}, \mathrm{emp}}(z)$ and $\mathrm{C}_{\mathrm{r}, \mathrm{emp}}(z)$ are the values taken from the SDSS data base, as well as the redshift $z$. The calculated Petrosian radii (both in $\mathrm{kpc}$ and in arcsec) and concentration

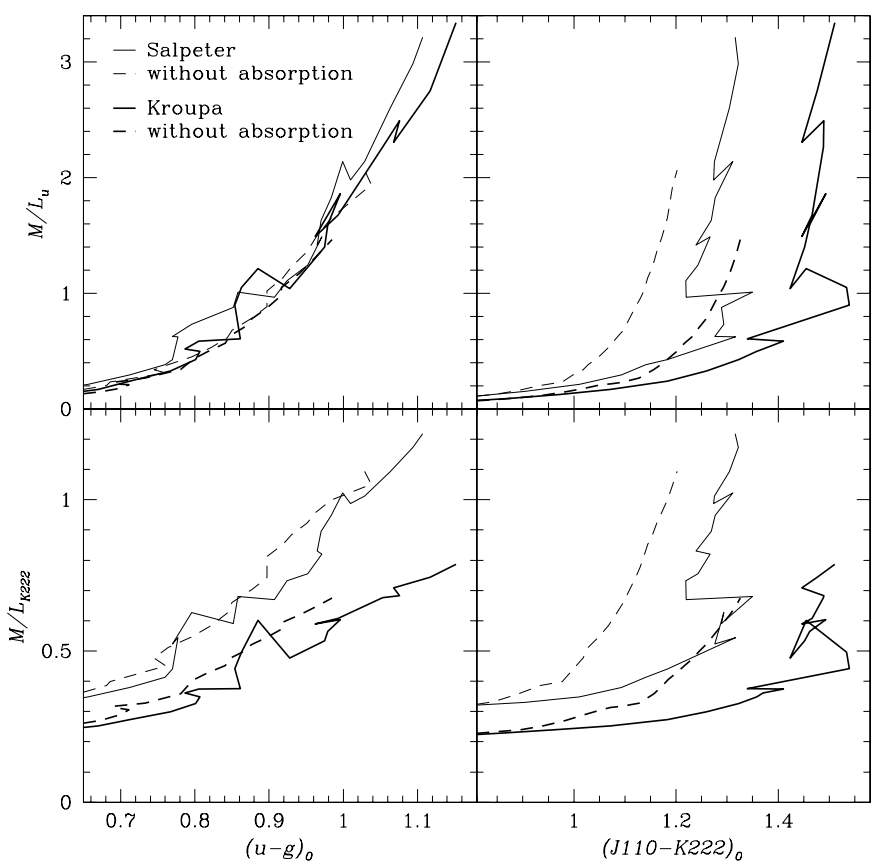

Fig. 11. Colour- $M / L$ relations of the two models in different passbands, including and omitting absorption.

indices are given for the Kroupa model in Tables D.1 to D.3, as they could be useful to identify Milky Way-type galaxies or progenitors at high redshift. At the first time step $(0.5 \mathrm{Gyr}$, redshift 9.5116), however, these values still suffer from the initial border conditions of the model, and cannot be used. The concentration indices could only be calculated from redshift 1.5915 on.

The apparent $u$ band magnitudes including their error bars of the subsamples are plotted as a function of redshift in Fig. 14. The thick dashed and dotted lines show the evolution of the corresponding models (dashed: face-on, dotted: edge-on). The thin lines show the respective other views for the same models (thus the thick lines in the upper panels correspond to the thin lines in the lower panels, and vice-versa), to show the differences in brightness between the two viewing angles. The models are only shown until the second-last time step at redshift 0.0369 , as the magnitudes at redshift 0 depend on the actual distance, which is unknown. The distance modulus $m-M$ of 25 used in Tables A.5 to A.8 is only a convention, whereas in reality the distance modulus at zero redshift depends upon the actual distance and Eq. (2) is not valid.

As can be seen from Fig. 14, the models represent the $u$ magnitudes of the empirical samples well, and they even reproduce the brightness differences between the face-on and the edge-on views.

Figure 15 shows the same galaxies and models as Fig. 14, but in the $r$ band. Here too, the agreement is good, although one could argue that the magnitudes of the Salpeter model are a bit too faint.

\subsection{Apparent colours}

Let us move on to apparent colours. Figure 16 shows the redshift evolution of apparent $u-g$ and $J 110-K 222$, the different line types and widths having the same meaning as in Fig. 13. The figure is only shown out to redshift 5.9, due to the above-mentioned imprecisions at redshift 9.5116. In $u-g$, they might even affect the data point at redshift 5.6177. In $u-g$, we again see our "IMF 


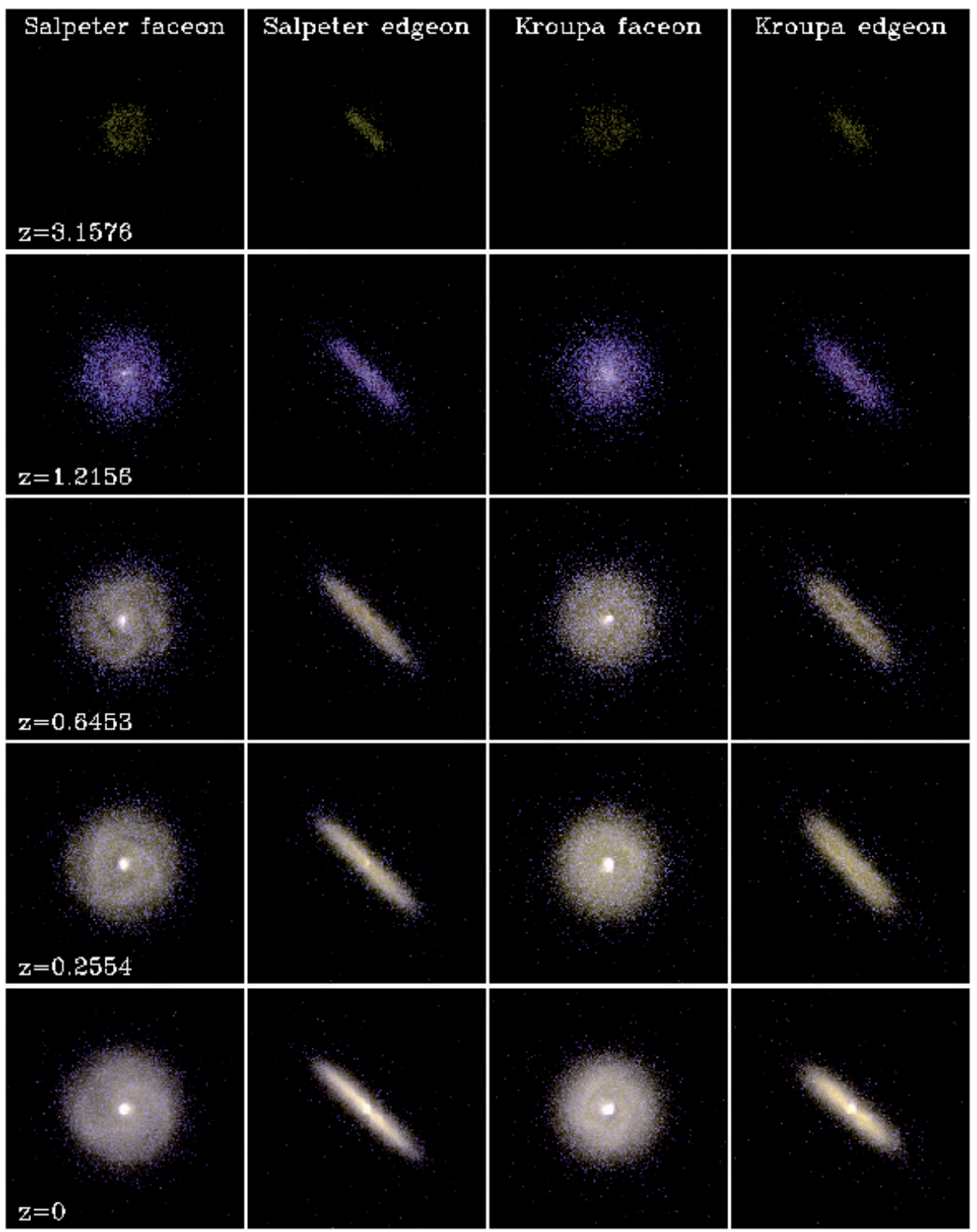

Fig. 12. Calculated apparent urz images of the Salpeter and Kroupa models, face-on and edgeon, both in the same magnitude scaling (corrected for distance modulus), where the calculated $u$ distribution makes up for the blue portion of the composite image, the $r$ distribution for the yellow portion, and the $z$ distribution for the red portion. The images correspond to the images in Fig. 3. degeneracy", the fact that the higher absorption in the Kroupa model compensates the bluer colour caused by the higher SFR. This leaves the $u-g$ differences between the two models at a level of at most $0.05 \mathrm{mag}$, which is undetectable given the much larger variations this colour shows during the evolution. At best, the differences could be seen in $J 110-K 222$, where they amount to $\sim 0.2 \mathrm{mag}$ (edge-on up to $0.4 \mathrm{mag}$ ), which is a significant fraction of the variation of this colour after $2.5 \mathrm{Gyr}$. ( $\sim 0.7 \mathrm{mag})$. Let us see how the models compare to the empirical data. In Figs. 17 to 19 , we see the $u-g, r-i$, and $r-z$ colours of the two models, face-on and edge-on, as a function of redshift in the same coding as in Fig. 14. Overplotted are the colours including error bars of the four subsamples of galaxies selected using Eq. (3), so the models are only compared to data from galaxies of the same types (angular sizes, concentration parameters, and viewing angles).

Obviously, the model colours are too blue by about 0.3 to $0.4 \mathrm{mag}$ in $u-g$, by about $0.1 \mathrm{mag}$ in $r-i$, and more or less in the right colour range for $r-z$. Also, the trends with redshift are not always well reproduced in the latter two colours. This could be an indication, that hierarchical-based accretion histories may induce a delayed galaxy formation, resulting in too blue colours especially at high redshift. It looks like SDSS galaxies on average have an earlier Hubble type star formation history than the Milky Way type model galaxy. This argument is also supported by the fact, that the monolithic collapse model in Westera et al. (2002b) produces colours in better agreement with the data. In spite of these systematic deviations, the models do seem to reproduce relative properties, i.e. the effect of galaxy orientation on integrated colours, as can be seen from the fact, that they nicely reproduce the shift between face-on - and edgeon colours, thereby underlining the usefulness of the corrections given in Tables C. 1 to C.6. This is why we believe that the results we found comparing the models with different IMFs are also realistic. We thus conclude that it is difficult to make statements about the stellar IMF from integrated colours or magnitudes of galaxies. Our best bet for this purpose are infrared colours, i.e. $J 110-K 222$.

\section{Summary and conclusions}

In this work, we use chemo-dynamical models to investigate the influence of the Initial Mass Function (IMF) on the evolution of a Milky Way-type disk galaxy, in particular of its colours.

For this purpose, we developed two chemodynamical models of such a galaxy with the same boundary conditions, but using 


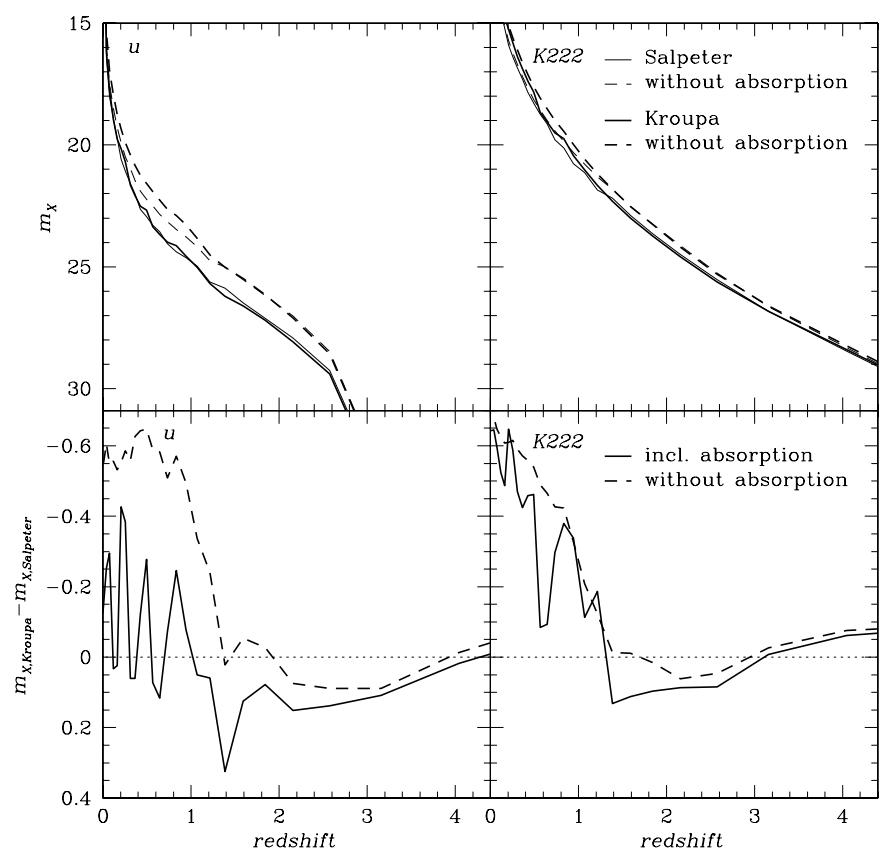

Fig. 13. Top panels: apparent $u$ and $K 222$ magnitude evolution of the Salpeter (thin) and the Kroupa (thick) models as a function of redshift, both including (solid) and omitting (dashed) gas absorption. Bottom panels: differences in $u$ and $K 222$ magnitudes between the Salpeter and the Kroupa models, both including (solid) and omitting (dashed) gas absorption. The thin dotted lines show the zero level.

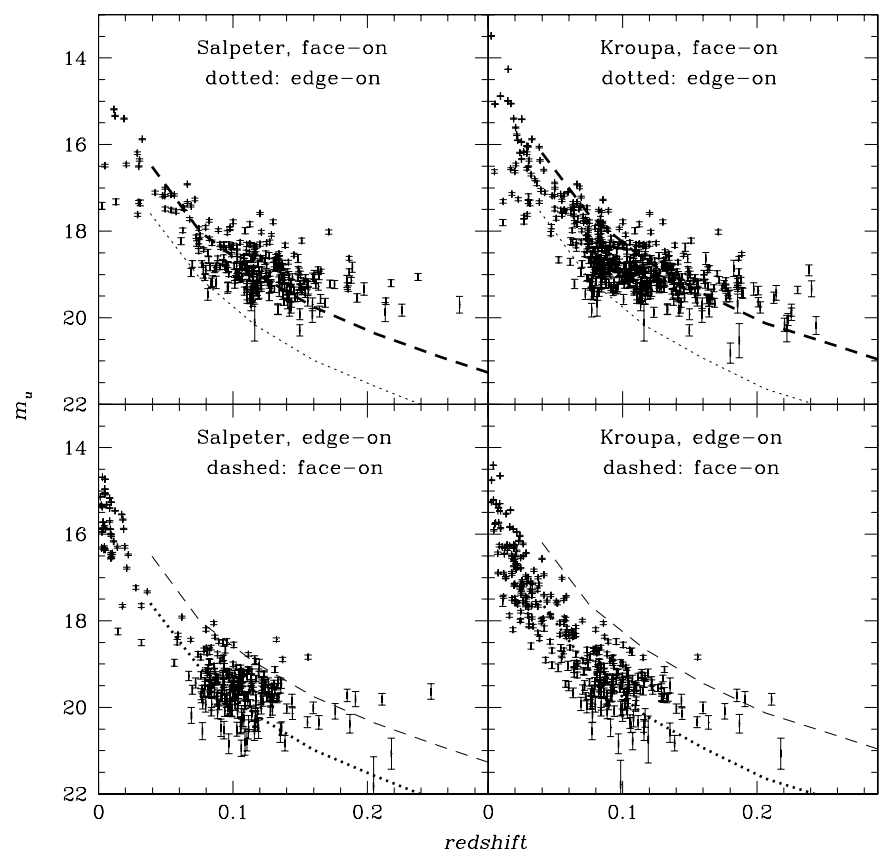

Fig. 14. Apparent $u$ magnitudes of the Salpeter and Kroupa models as seen face-on (dashed) and edge-on (dotted) as a function of redshift. Overlaid are the SDSS DR4 data with their observational errors of galaxies that have similar sizes, concentration parameters, and orientation as the models (for a more precise description, see the text).

different IMFs: Salpeter and Kroupa, which differ in their lowto-high mass stars ratios. The Kroupa model, having a higher fraction of high mass stars, begins with a lower SFR than the Salpeter model, but from 5 Gyr on, this reverses. The Kroupa model also has a higher gas density and metallicity than the Salpeter model at all ages.

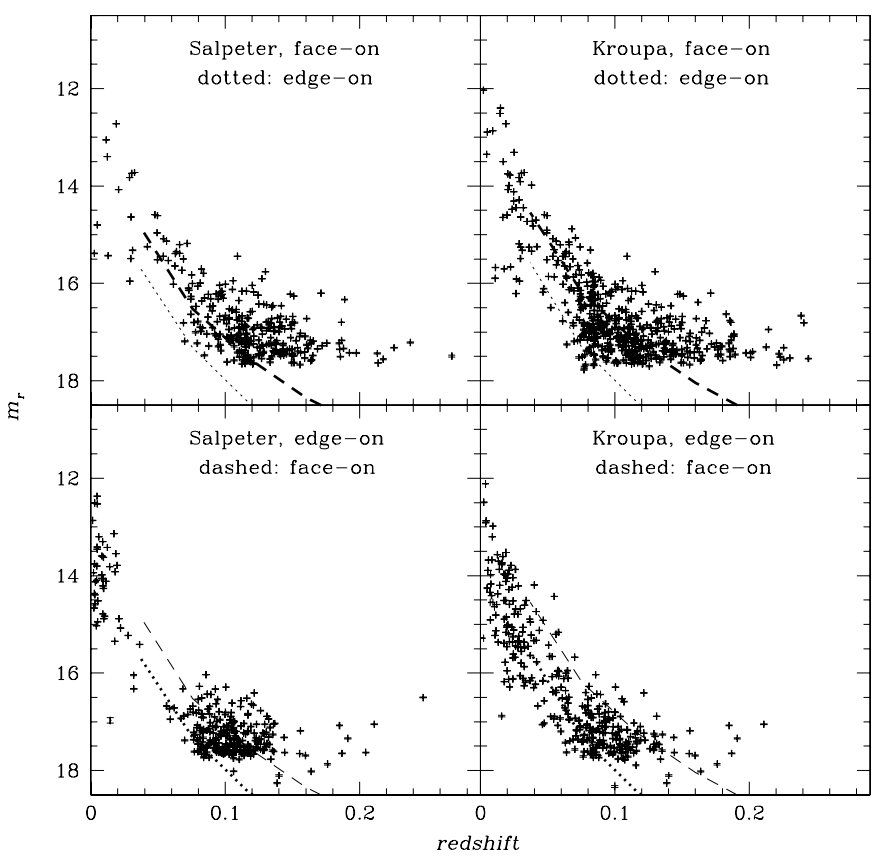

Fig. 15. Like Fig. 14, but for the $r$ band.

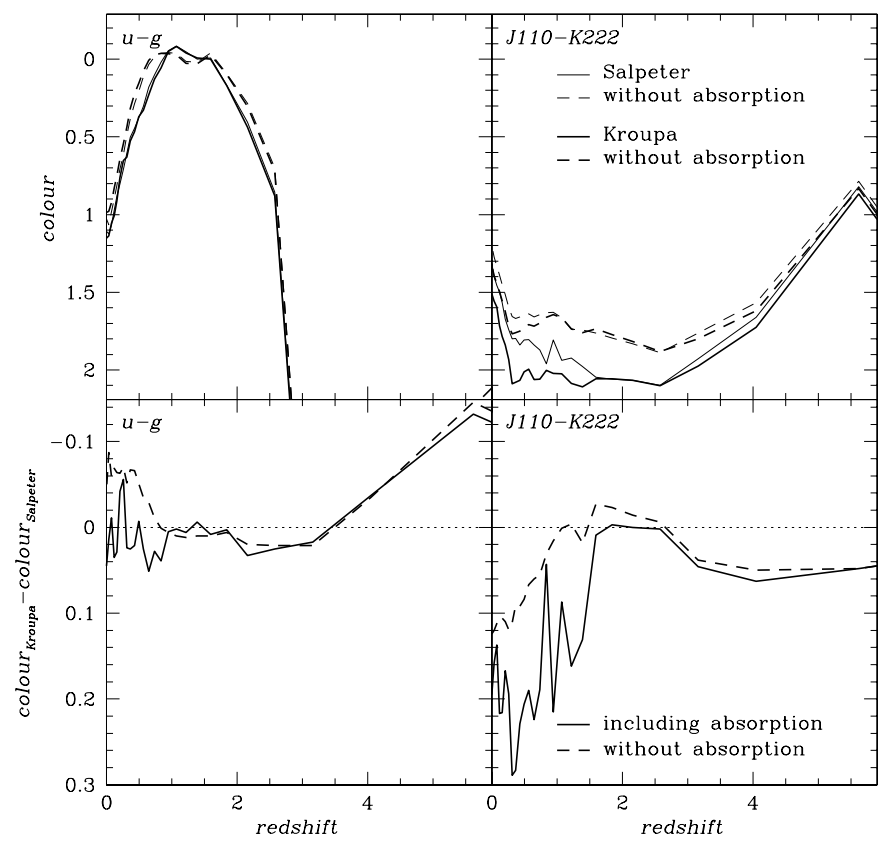

Fig. 16. Top panels: apparent $u-g$ and $J 110-K 222$ colour evolution of the Salpeter (thin) and the Kroupa (thick) models as a function of redshift, both including (solid) and omitting (dashed) gas absorption. Bottom panels: differences in the $u-g$ and $J 110-K 222$ colours between the Salpeter and the Kroupa models, both including (solid) and omitting (dashed) gas absorption. The thin dotted lines show the zero level.

With these two models, we performed a spectral analysis, evaluated with a state of the art evolutionary code and spectral library. The programme transforming the models into spectral properties takes into account the three-dimensional distribution of the stars and the interstellar matter. It includes internal gas absorption and re-emission and is also able to include foreground reddening. We obtain two-dimensional HST (WFPC2 and NICMOS), and SDSS ugriz images of the model galaxies, giving intrinsic and apparent magnitudes and colours in up to $320 \times 320$ pixels. We also obtain intrinsic and apparent 


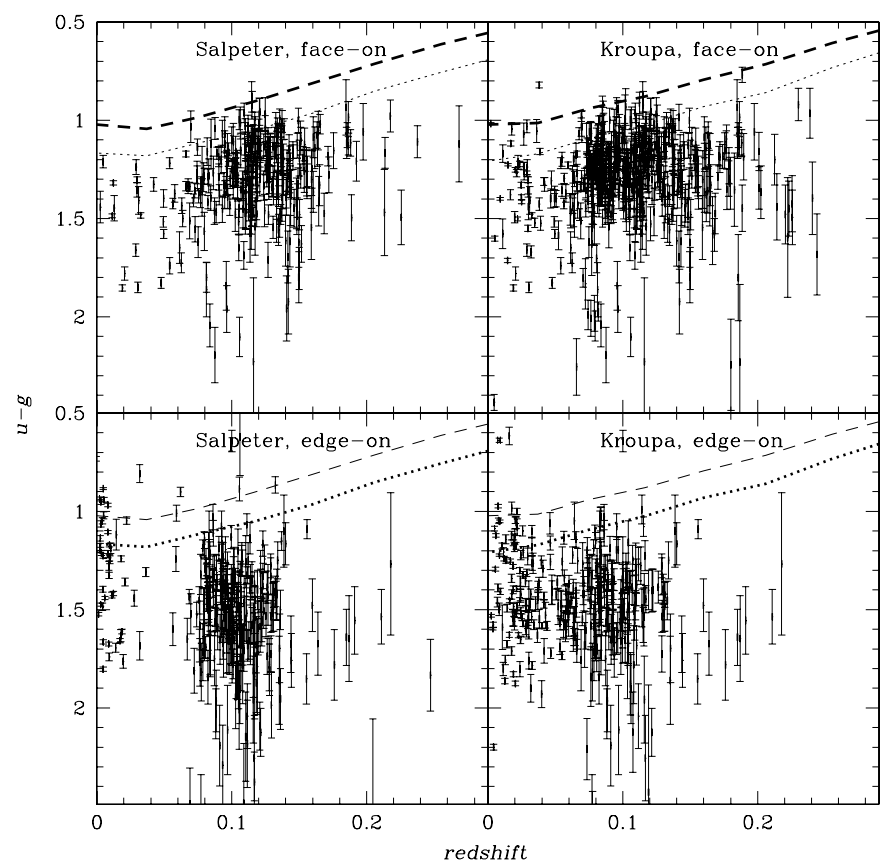

Fig. 17. Apparent $u-g$ colours of the Salpeter and Kroupa models as seen face-on (dashed) and edge-on (dotted) as a function of redshift. Overlaid are the SDSS DR4 data with their observational errors of galaxies that have similar sizes, concentration parameters, and orientation as the models (for a more precise description, see the text).

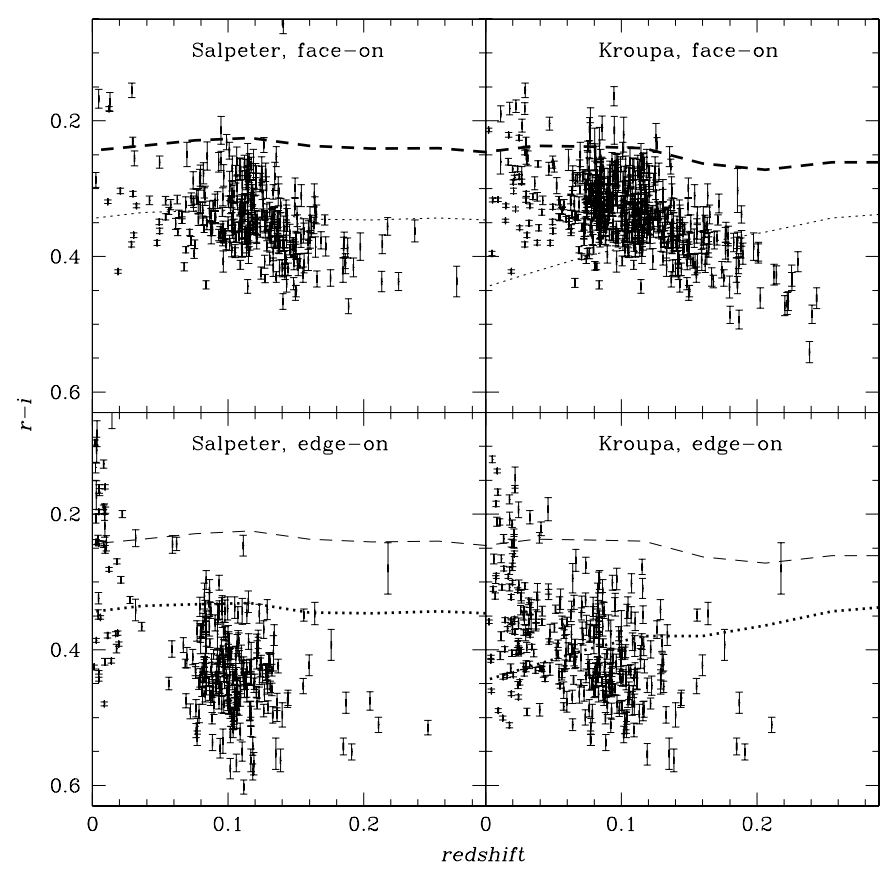

Fig. 18. Like Fig. 17, but for $r-i$.

integrated spectra and colours of the model galaxies. All of these quantities were calculated with a time resolution of $0.5 \mathrm{Gyr}$. The programme is able to view the model galaxies from different angles (diagonally, face-on, and edge-on), which allows to analyse orientation effects on the spectral properties. Furthermore, by recalculating the models artificially omitting the gas absorption, we could disentangle absorption effects from other effects. We provide photometric absorption and inclination corrections

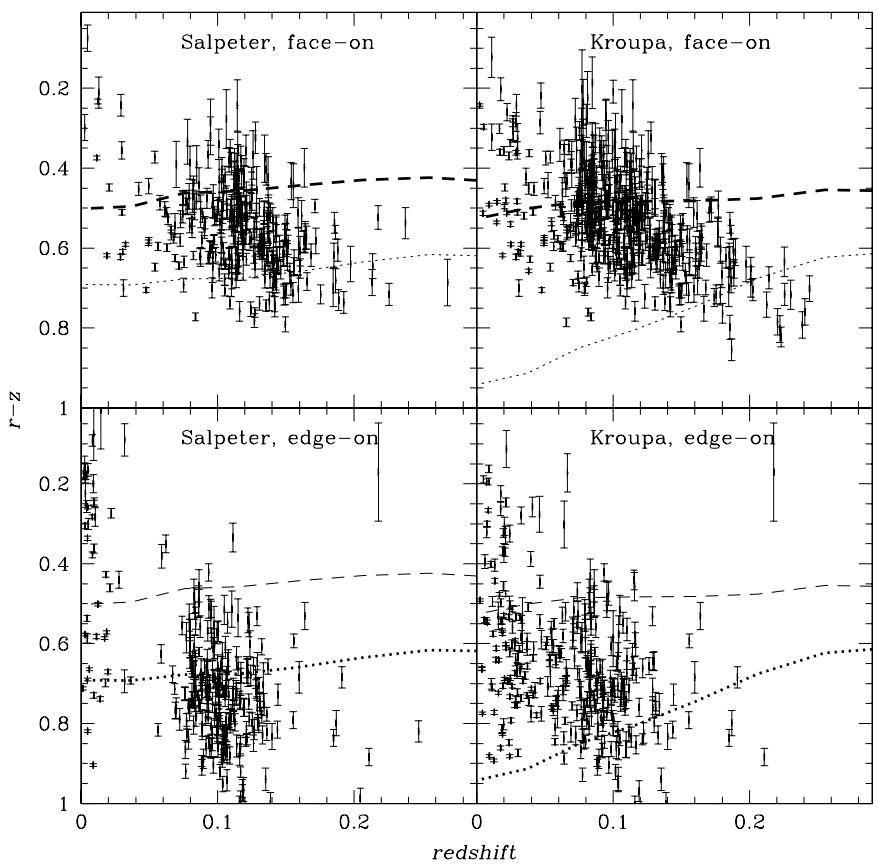

Fig. 19. Like Fig. 17, but for $r-z$.

in the SDSS ugriz and the HST WFPC2 and NICMOS systems (Tables C.1 to C.6).

We find, that the effect of the IMF on the internal gas absorption is larger than its effect on the light from the stellar content. However, the two effects work in the opposite sense (An IMF with more high mass stars leads to brighter and bluer stellar light, but also to more interstellar dust and thus to more absorption), causing a kind of "IMF degeneracy". The most likely wavelength region, in which to detect IMF effects is the infrared (i.e. $J H K)$. Here, the differences between the two models amount to $\sim 0.5 \mathrm{mag}$ in $K 222$, and to $\sim 0.2 \mathrm{mag}$ in $J 110-K 222$.

The effects of inclination on a galaxy's magnitudes and colours, on the other hand, are larger than the ones due to the IMF. Seeing a galaxy edge-on instead of face-on or diagonally can make it appear up to 1.5 mag fainter in $u$, or 1 mag in $z$, or 0.6 mag redder in $J 110-K 222$.

A comparison of the calculated integrated model magnitudes and colours with SDSS data partly shows good agreement, especially in the $u$ and $g$ magnitudes, and the riz colours. There are some systematic deviations in the UV colours, indicating that SDSS galaxies might have on average an earlier Hubble type star formation history than the model galaxy, which is more like the Milky Way. On the other hand, the relative tendencies in these colours are well reproduced (i.e. the shift between face-on and edge-on colours of galaxies of the same type). We conclude from this, that the theoretical results presented in the two previous paragraphs should hold true in practice as well.

As a side study, we verify the assumption by Bell \& de Jong (2001), that the combined absorption and reddening effects of dust do not significantly alter the colour- $M / L$ relations for galaxy models, and find this assumption only to be true for colours involving passbands in the bluer wavelength ranges (bluewards of the $I$ band).

Acknowledgements. This work was supported by the Swiss National Science Foundation. 


\section{References}

Adelman-McCarthy, J. K., et al. 2005, ApJS, 162, 38

Barnes, J., \& Efstathiou, G. 1987, ApJ, 319, 575

Bell, E. F., \& de Jong, R. S. 2001, ApJ, 550, 212

Blanton, M. R., Dalcanton, J., Eisenstein, D., et al. 2001, AJ, 121, 2358

Bruzual, A. G., \& Charlot, S. 1993, ApJ, 405, 538

Bruzual, A. G., \& Charlot, S. 2003, MNRAS, 344, 1000

Bullock, J. S., Dekel, A., Kolatt, T. S., et al. 2001, ApJ, 555, 240

Carigi, L., Colín, P., \& Peimbert, M. 1999, ApJ, 514, 787

Carroll, S. M., Press, W. H., \& Turner, E. L. 1992, ARA\&A, 30, 499

Chabrier, G. 2001, ApJ, 554, 1274

Chabrier, G. 2002, ApJ, 567, 304

Chabrier, G. 2003, PASP, 115, 763

Charlot, S., \& Bruzual, A. G. 1991, ApJ, 367, 126

Eggen, O. J., Lynden-Bell, D., \& Sandage, A. R. 1962, ApJ, 136, 748

Fagotto, F., Bressan, A., Bertelli, G., \& Chiosi, C. 1994, A\&AS, 105, 39

Ferraro, F. R., Carretta, E., Bragaglia, A., Renzini, A., \& Ortolani, S. 1997, MNRAS, 286, 1012

Fluks, M. A., Plez, B., The, P. S., et al. 1994, A\&AS, 105, 311

Girardi, L., Bressan, A., Chiosi, C., Bertelli, G., \& Nasi, E. 1996, A\&AS, 117, 113

Girardi, L., Bressan, A., Bertelli, G., \& Chiosi, C. 2000, A\&AS, 141, 371, Padova 2000, isochrones

Gould, A., Bahcall, J. N., \& Flynn, C. 1997, ApJ, 482, 913

Gould, A., Flynn, C., \& Bahcall, J. N. 1998, ApJ, 503, 798

Kautsch, S. J., Grebel, E. K., Barazza, F. D., \& Gallagher, J. S., III 2006, A\&A, 445,765

Kennicutt, R. C. 1983, ApJ, 272, 54

Kroupa, P. 1998, The Stellar Mass Function (invited review) in Brown Dwarfs and Extrasolar Planets, ed. R. Rebolo, L. Eduardo Martin, \& M. R. Zapatero Osorio (San Francisco: ASP), ASP Conf. Ser., 134, 483

Kroupa, P. 2001, MNRAS, 322, 231

Kroupa, P. 2002, Science, 295, 82

Kroupa, P., Tout, C. A., \& Gilmore, G. 1993, MNRAS, 262, 545
Larson, R. B. 1998, MNRAS, 301, 569

Leitherer, C., Schaerer, D., Goldader, J. D., et al. 1999, ApJS, 123, 3

Lequeux, J. 1979, A\&AS, 80, 35

Lerner, M. S., Sundin, M., \& Thomasson, M. 1999, A\&A, 344, 483

Madau, P. 1995, ApJ, 441, 18

Miller, G. E., \& Scalo, J. M. 1979, ApJS, 41, 513

Petrosian, V. 1976, ApJ, 209, L1

Piotto, G., Cool, A. M., \& King, I. R. 1997, AJ, 113, 1345

Piotto, G., \& Zoccali, M. 1999, A\&A, 345, 485

Portinari, L., Sommer-Larsen, J., \& Tantalo, R. 2004, MNRAS, 347, 691

Quillen, A. C., \& Yukita, M. 2001, AJ, 121, 2095

Reid, I. N., \& Gizis, J. E. 1997, AJ, 113, 2246

Salpeter, E. E. 1955, ApJ, 121, 161

Samland, M., \& Gerhard, O. E. 2003, A\&A, 399, 961

Scalo, J. M. 1986, The initial mass function of massive stars in galaxies Empirical evidence, in Luminous Stars and Associations in Galaxies, IAU Symp., 116, 451

Scalo, J. M. 1998, The IMF Revisited: A Case for Variations, in The Stellar Initial Mass Function, 38th Herstmonceux Conference, ed. G. Gilmore, \& D. Howell (San Francisco: ASP), ASP Conf. Ser., 142, 201

Stephens, A. W., Frogel, J. A., Ortolani, S., et al. 2000, AJ, 119, 419

van den Bosch, F. C. 2002, MNRAS, 331, 98

Wechsler, R. H., Bullock, J. S., Primack, J. R., Kravtsov, A. V., \& Dekel, A. 2002, ApJ, 568, 52

Westera, P. 2001, The BaSeL 3.1 models: Metallicity calibration of a theoretical stellar spectral library and its application to chemo-dynamical galaxy models, Ph.D. Thesis, Univ. of Basel, 378

Westera, P., Lejeune, T., Buser, R., Cuisinier, F., \& Bruzual, A. G. 2002a, A\&A, 381,524

Westera, P., Samland, M., Buser, R., \& Gerhard, O. E. 2002b, A\&A, 389, 761

Wilkinson, M. I., \& Evans, M. W. 1999, MNRAS, 310, 645

Yasuda, N., Fukugita, M., Narayanan, V. K., et al. 2001, AJ, 122, 1104

York, D. G., Adelman, J., Anderson, J. E., Jr., et al. 2000, AJ, 120, 1579

Zoccali, M., Cassisi, S., Frogel, J. A., et al. 2000, ApJ, 530, 418 
P. Westera et al.: Initial mass function effects on the colour evolution of disk galaxies, Online Material $p 1$

\section{Online Material}


P. Westera et al.: Initial mass function effects on the colour evolution of disk galaxies, Online Material p 2

\section{Appendix A: Intrinsic magnitudes}

Table A.1. Intrinsic integrated SDSS magnitudes of the Salpeter model.

\begin{tabular}{|c|c|c|c|c|c|c|c|}
\hline$\overline{t[\mathrm{Gyr}]}$ & $\overline{M_{\text {Bol }}}$ & $\overline{\bar{M} M_{V_{j}}}$ & $\overline{M_{u}}$ & $\overline{M_{g}}$ & $\overline{M_{r}}$ & $\overline{M_{i}}$ & $M_{z}$ \\
\hline 0.5 & 15.586 & -12.621 & -13.207 & -12.918 & -12.430 & -12.171 & -11.957 \\
\hline 1.0 & & -17.749 & 17.563 & -17.844 & -17.706 & -17.643 & -17.583 \\
\hline 1.5 & & -19 . & -18.736 & -19.187 & -19.157 & -19.147 & -19.14 \\
\hline 2.0 & & -19.964 & -19.403 & -19.944 & -19.986 & -20.013 & -20.04 \\
\hline 2.5 & & -20.505 & -19.843 & -20.449 & -20.548 & -20.609 & -20.682 \\
\hline 3.0 & & -20 & -20.114 & -20.770 & -20.916 & -21.008 & 117 \\
\hline 3.5 & 2.004 & -21.067 & -20.242 & -20.954 & -21 & 261 & 97 \\
\hline 4.0 & 2.095 & -21.208 & -2 & 70 & 01 & 37 & \\
\hline 4. & 55 & 39 & -2 & 48 & -2 & 14 & 64 \\
\hline 5. & -2 & 11 & -2 & -21 & -2 & 47 & -2 \\
\hline 5. & -2 & 38 & -2 & -2 & -2 & 59 & -2 \\
\hline 6. & 2 & 11 & -2 & -2 & -2 & -2 & -2 \\
\hline 6. & -22 . & 44 & -20 & -21 & -21 & -2 & 991 \\
\hline 7. & -22.266 & -21 & -2 & -21 & 30 & -21 & -21.898 \\
\hline 7.5 & -2 & 21 & -20 . & -21 & -21.764 & -2 & -22.148 \\
\hline $8 .($ & -2 & -21 & -20 & -21 & -21.735 & -21 & -22.135 \\
\hline 8. & 61 & -2 & -2 & -2 & -21 & -21 & -22.139 \\
\hline 9.0 & 70 & -21 & -20 & -21 & -21.638 & -21 & -22.083 \\
\hline 9.5 & & & -20 & -21.317 & -21 & -21 & -22.160 \\
\hline 10.0 & -22 & -21 & -20 & -21.223 & -21.651 & -21 & -22.095 \\
\hline 5 & 12 & 28 & -20 & -21 & -21.604 & -21.833 & -22.066 \\
\hline & -22 & -2 & -20 & -21 & -21 & -21.748 & -21.990 \\
\hline & -22 & -21 & -20 & -21 & -21 & -21.870 & -22.106 \\
\hline & -22 & & -20 & & & & 09 \\
\hline & -22 & -21 & -19 & -21 & -21 & -2 & -22 \\
\hline & -22.022 & 28 & -19 & -20 & 32 & -21 & -21 \\
\hline .5 & -21.989 & -21.193 & -19.755 & -20.862 & -21.404 & -21.670 & -21.935 \\
\hline
\end{tabular}

Table A.2. Intrinsic integrated HST magnitudes of the Salpeter model.

\begin{tabular}{|c|c|c|c|c|c|c|c|c|c|}
\hline Gyr] & $\overline{M_{U 336}}$ & $M_{B 439}$ & $M_{V 555}$ & $M_{V 606}$ & $M_{R 675}$ & $\overline{M_{I 814}}$ & $\overline{M_{J 110}}$ & $\overline{M_{H 160}}$ & $M_{K 2}$ \\
\hline 0.5 & -14. & & -12.70 & 2.65 & 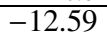 & -12.55 & -12.48 & 2.49 & -12.52 \\
\hline 1. & -18. & -11 & $-1 /$ & $-1 /$ & 0 & 10 & -18 & -1 & -18 . \\
\hline 1. & -19 & & & - & & & & & 20 \\
\hline 2.0 & -20 & -19 & -19 & -20 & -20 & 20.52 & -2( & -2 & 21 \\
\hline 2.5 & -20.94 & -20 & -20.49 & -20.63 & -20 & & -2 & -2 & 22. \\
\hline 3.0 & -21 & & & -20 & -21 & & & -2 & -22.87 \\
\hline 3.5 & & -2 & -2 & -2 & -2 & -2 & -2 & -2 & 3.26 \\
\hline 4.1 & -2 & -20 & -2 & & -2 & -2 & -2 & & 51 \\
\hline 4.5 & -2 & -21 & -2 & -2 & -2 & 9 & -2 & 37 & 13 \\
\hline $5 .($ & -2 & -21 & -2 & -2 & -2 & -2 & -2 & 29 & -2 \\
\hline 5. & -21. & -21 & -2 & -2 & -2 & -2 & -2 & 6 & - \\
\hline 6. & -21. & -2 & -21 & -2 & -2 & -2 & -2 & 18 & -2 \\
\hline 6.5 & -21. & -2 & -21 & -2 & -2 & -22 & -22 & 73 & -2 \\
\hline 7. & -21.4 & -20 & -2 & -2 & -2 & -2 & -2 & 64 & -24.07 \\
\hline 7. & -21.1 & -2 & -2 & -2 & -22.12 & -22 & -22.96 & -23.82 & -24.18 \\
\hline 8. & 2 & -21 & -21 & -21 & -22 & -2 & -22.95 & -23.80 & -24.17 \\
\hline 8. & -21. & -2 & -2 & -2 & -2 & 54 & -2 & 83 & -24.20 \\
\hline 9.0 & -21. & -2 & -2 & -21. & -2 & -2 & -2 & 80 & -24.18 \\
\hline 9.5 & & & -2 & -2 & -2 & -2 & -22.98 & -23 & -24.22 \\
\hline & & -2 & -2 & -2 & -22 & -2 & -2 & -23.80 & -24.19 \\
\hline & & -20 & -2 & -2 & -21.98 & -22.46 & -22.90 & -23.78 & -24.18 \\
\hline & & -20 & & & -21.89 & -22.38 & -22.83 & -23.73 & -24.14 \\
\hline & & -20 & -2 & -2 & -22.02 & -22 & -22.94 & -23.82 & -24.21 \\
\hline & & -2 & & & -22 & -22.50 & -22.95 & -23 & -24.22 \\
\hline & & -20 & & & -21 & -22 & -2 & & -2 \\
\hline & & -20 & -21 & -2 & -21 & -22 & -22 & -2 & -24.12 \\
\hline & & -20 & -21 & -21 & -21 & -22 & -22 & -23.69 & -2 \\
\hline
\end{tabular}


P. Westera et al.: Initial mass function effects on the colour evolution of disk galaxies, Online Material p 3

Table A.3. Intrinsic integrated SDSS magnitudes of the Kroupa model.

\begin{tabular}{rrrrrrrr}
\hline \hline$t[\mathrm{Gyr}]$ & $M_{\text {Bol }}$ & $M_{V_{j}}$ & $M_{u}$ & $M_{g}$ & $M_{r}$ & $M_{i}$ & $M_{z}$ \\
\hline 0.5 & -15.823 & -13.180 & -13.541 & -13.414 & -12.994 & -12.714 & -12.490 \\
1.0 & -19.526 & -17.907 & -17.659 & -17.993 & -17.839 & -17.751 & -17.705 \\
1.5 & -20.522 & -19.227 & -18.769 & -19.252 & -19.203 & -19.179 & -19.189 \\
2.0 & -21.098 & -19.968 & -19.380 & -19.950 & -19.975 & -19.996 & -20.047 \\
2.5 & -21.482 & -20.434 & -19.757 & -20.380 & -20.466 & -20.528 & -20.618 \\
3.0 & -21.762 & -20.777 & -20.025 & -20.695 & -20.830 & -20.924 & -21.046 \\
3.5 & -21.937 & -20.966 & -20.147 & -20.859 & -21.039 & -21.161 & -21.315 \\
4.0 & -22.007 & -21.083 & -20.184 & -20.951 & -21.173 & -21.318 & -21.493 \\
4.5 & -22.056 & -21.145 & -20.189 & -20.990 & -21.252 & -21.419 & -21.614 \\
5.0 & -22.172 & -21.249 & -20.277 & -21.084 & -21.364 & -21.539 & -21.745 \\
5.5 & -22.365 & -21.417 & -20.465 & -21.252 & -21.533 & -21.710 & -21.922 \\
6.0 & -22.477 & -21.530 & -20.553 & -21.358 & -21.652 & -21.837 & -22.059 \\
6.5 & -22.646 & -21.780 & -20.727 & -21.588 & -21.913 & -22.105 & -22.334 \\
7.0 & -22.447 & -21.497 & -20.446 & -21.300 & -21.635 & -21.838 & -22.079 \\
7.5 & -22.424 & -21.474 & -20.400 & -21.263 & -21.622 & -21.838 & -22.087 \\
8.0 & -22.375 & -21.460 & -20.353 & -21.238 & -21.614 & -21.834 & -22.080 \\
8.5 & -22.693 & -21.789 & -20.618 & -21.546 & -21.958 & -22.183 & -22.444 \\
9.0 & -22.542 & -21.630 & -20.380 & -21.355 & -21.819 & -22.075 & -22.361 \\
9.5 & -22.523 & -21.582 & -20.315 & -21.294 & -21.781 & -22.052 & -22.354 \\
10.0 & -22.466 & -21.508 & -20.214 & -21.210 & -21.714 & -21.995 & -22.307 \\
10.5 & -22.671 & -21.747 & -20.512 & -21.474 & -21.936 & -22.179 & -22.459 \\
11.0 & -22.639 & -21.719 & -20.440 & -21.433 & -21.916 & -22.166 & -22.454 \\
11.5 & -22.464 & -21.535 & -20.158 & -21.211 & -21.755 & -22.038 & -22.350 \\
12.0 & -22.461 & -21.514 & -20.097 & -21.173 & -21.745 & -22.042 & -22.369 \\
12.5 & -22.527 & -21.619 & -20.220 & -21.288 & -21.842 & -22.118 & -22.426 \\
13.0 & -22.440 & -21.529 & -20.062 & -21.179 & -21.763 & -22.052 & -22.369 \\
13.5 & -22.344 & -21.405 & -19.880 & -21.032 & -21.652 & -21.958 & -22.292 \\
\hline
\end{tabular}

Table A.4. Intrinsic integrated HST magnitudes of the Kroupa model.

\begin{tabular}{|c|c|c|c|c|c|c|c|c|c|}
\hline$\overline{\mathrm{G}} \overline{\mathrm{Gyr}]}$ & $\overline{M_{U 336}}$ & $M_{B 439}$ & $\bar{M}_{V 555}$ & $\overline{M_{V 606}}$ & $\overline{M_{R 675}}$ & $M_{I 814}$ & $\bar{M}_{J 110}$ & $\bar{M}_{H 160}$ & $\overline{\overline{M_{K 2}}}$ \\
\hline 0.5 & -14.80 & 3.39 & 3.24 & -13.20 & -13.14 & -13.09 & -13.02 & -13.04 & -13.07 \\
\hline 1.0 & -1 & -1 & & 8 & & 2 & $-1 \xi$ & 8 & -18 \\
\hline 1.5 & -19. & & & & & -1 & -1 & & -20 \\
\hline 2.0 & -20 & & & & & -2 & -2 & & 53 \\
\hline 2.5 & -20 & -20 & 42 & -20 & -2 & -2 & -2 & & 33 \\
\hline 3.0 & -21 & -20 & -20 & -2 & -2 & -2 & -2 & 57 & -22.89 \\
\hline 3. & -21 & -2 & -2 & -2 & -2 & -2 & 11 & 94 & -2 \\
\hline 4. & -21. & -2 & -2 & -2 & -2 & -1 & 31 & 18 & - \\
\hline 4.8 & -21 & -2 & -2 & -2 & -2 & - & - & 36 & - \\
\hline 5. & -21. & -20 & -2 & -2 & -2 & -2 & 59 & 52 & -2 \\
\hline 5. & -21.53 & -2 & -2 & - & -2 & -2 & -2 & 71 & -2 \\
\hline 6. & -2 & -21.14 & -21.48 & -21.69 & -22.01 & -22.45 & -22.92 & 88 & -24.33 \\
\hline 6. & -2 & -21 & -2 & -2 & -22 & -22.73 & -23.19 & 11 & -24.53 \\
\hline 7. & -2 & -21 & -2 & -2 & -2 & 46 & -2 & 99 & -24.50 \\
\hline 7 & $-2+2$ & -2 & -2 & -2 & 9 & 47 & 97 & - & -24.50 \\
\hline 8. & & -2 & -2 & & -2 & -2 & -2 & & -24.41 \\
\hline ס. & & -2 & -2 & -2 & -22 & -22 & -2 & -2 & -24.76 \\
\hline 9.0 & -2 & -2 & -2 & -2 & -2 & -22.13 & -23.27 & -2 & -24.72 \\
\hline 9.5 & -2 & -2 & & -2 & -2 & -22.72 & -23.27 & 27 & -24.73 \\
\hline & -2 & -2 & -2 & -2 & -2 & -22 & -23.23 & -24.25 & -24.73 \\
\hline & -2 & -2 & -2 & -2 & -2 & -2 & -2 & -2 & -24.81 \\
\hline & -2 & -2 & & & -2 & -2 & -2 & & -24.83 \\
\hline & & -20 & -21 & -2 & -2 & -22.71 & -2 & 28 & $-24 . / 0$ \\
\hline & -21 & -20 & -2 & -2 & -2 & -22.72 & -2 & -2 & -24.79 \\
\hline & & -2 & -2 & & -2 & -22.79 & -2 & -2 & -24.79 \\
\hline & & -2 & -2 & -2 & -2 & -22 & -23 & -2 & -24.77 \\
\hline & -2 & & & & -2 & -22.64 & -2 & -24.25 & -24.7 \\
\hline
\end{tabular}


Table A.5. Apparent integrated SDSS magnitudes of the Salpeter model.

\begin{tabular}{|c|c|c|c|c|c|c|c|c|c|}
\hline$\overline{t[\mathrm{Gyr}]}$ & $\overline{\text { Redshift }}$ & $m-M$ & $m_{\mathrm{Bol}}$ & $m_{V_{j}}$ & $m_{u}$ & $m_{g}$ & $m_{r}$ & $m_{i}$ & $m_{z}$ \\
\hline 0.5 & 9.5116 & 49.953869 & 39.968 & 161.915 & 162.836 & 162.254 & 161.664 & 161.259 & 52.997 \\
\hline 1.0 & 5.6177 & 48.640133 & 33.535 & 39.367 & 59.169 & 56.694 & 36.545 & 34.365 & 32.700 \\
\hline 1.5 & 4.0444 & 47.801151 & 30.919 & 31.566 & 48.184 & 32.844 & 31.073 & 30.684 & 30.748 \\
\hline 2.0 & 3.1576 & 47.159698 & 29.177 & 29.381 & 34.035 & 29.857 & 29.311 & 29.372 & 29.479 \\
\hline 2.5 & 2.5758 & 46.626331 & 27.854 & 28.294 & 29.258 & 28.404 & 28.318 & 28.430 & 28.454 \\
\hline 3.0 & 2.1589 & 46.160126 & 26.821 & 27.528 & 27.922 & 27.517 & 27.579 & 27.665 & 27.552 \\
\hline 3.5 & 1.8422 & 45.738579 & 26.005 & 26.979 & 27.111 & 26.944 & 27.052 & 26.996 & 26.843 \\
\hline 4.0 & 1.5915 & 45.347797 & 25.320 & 26.548 & 26.479 & 26.487 & 26.562 & 26.385 & 26.177 \\
\hline 4.5 & 1.3868 & 44.978645 & 24.600 & 25.928 & 25.882 & 25.883 & 25.870 & 25.672 & 25.298 \\
\hline 5.0 & 1.2156 & 44.624722 & 24.237 & 25.664 & 25.623 & 25.670 & 25.548 & 25.368 & 24.711 \\
\hline 5.5 & 1.0696 & 44.281029 & 23.527 & 24.984 & 24.968 & 25.052 & 24.871 & 24.586 & 23.952 \\
\hline 6.0 & 0.9432 & 43.943302 & 23.092 & 24.549 & 24.631 & 24.685 & 24.422 & 23.904 & 23.449 \\
\hline 6.5 & 0.8322 & 43.607937 & 22.592 & 24.174 & 24.382 & 24.364 & 24.034 & 23.302 & 22.975 \\
\hline 7.0 & 0.7336 & 43.271690 & 22.198 & 23.744 & 24.058 & 23.957 & 23.527 & 22.787 & 22.564 \\
\hline 7.5 & 0.6453 & 42.931248 & 21.575 & 23.130 & 23.554 & 23.375 & 22.755 & 22.122 & 21.889 \\
\hline 8.0 & 0.5655 & 42.583164 & 21.170 & 22.682 & 23.287 & 22.984 & 22.194 & 21.681 & 21.447 \\
\hline 8.5 & 0.4928 & 42.223686 & 20.731 & 22.159 & 22.947 & 22.569 & 21.636 & 21.233 & 20.986 \\
\hline 9.0 & 0.4263 & 41.848011 & 20.347 & 21.657 & 22.644 & 22.199 & 21.176 & 20.853 & 20.594 \\
\hline 9.5 & 0.3650 & 41.450405 & 19.758 & 20.903 & 22.011 & 21.509 & 20.525 & 20.235 & 20.010 \\
\hline 10.0 & 0.3082 & 41.023094 & 19.317 & 20.355 & 21.580 & 20.974 & 20.071 & 19.777 & 19.564 \\
\hline 10.5 & 0.2554 & 40.554939 & 18.806 & 19.785 & 21.077 & 20.368 & 19.548 & 19.255 & 19.043 \\
\hline 11.0 & 0.2061 & 40.029339 & 18.292 & 19.237 & 20.578 & 19.776 & 19.023 & 18.729 & 18.505 \\
\hline 11.5 & 0.1599 & 39.418262 & 17.476 & 18.362 & 19.725 & 18.842 & 18.174 & 17.900 & 17.671 \\
\hline 12.0 & 0.1165 & 38.670574 & 16.667 & 17.526 & 18.921 & 17.948 & 17.337 & 17.081 & 16.828 \\
\hline 12.5 & 0.0756 & 37.670452 & 15.716 & 16.566 & 18.008 & 16.941 & 16.356 & 16.092 & 15.836 \\
\hline 13.0 & 0.0369 & 36.051773 & 14.108 & 14.932 & 16.398 & 15.274 & 14.713 & 14.456 & 14.192 \\
\hline 13.5 & 0.0000 & 24.999989 & 3.011 & 3.807 & 5.245 & 4.138 & 3.596 & 3.330 & 3.065 \\
\hline
\end{tabular}

Table A.6. Apparent integrated HST magnitudes of the Salpeter model.

\begin{tabular}{rrrrrrrrrr}
\hline \hline Redshift & $m_{U 336}$ & $m_{B 439}$ & $m_{V 555}$ & $m_{V 606}$ & $m_{R 675}$ & $m_{I 814}$ & $m_{J 110}$ & $m_{H 160}$ & $m_{K 222}$ \\
\hline 9.5116 & 161.8 & 162.6 & 162 & 161.7 & 161.2 & 58.57 & 39.79 & 36.96 & 36.71 \\
5.6177 & 57.42 & 59.80 & 39.05 & 36.33 & 34.78 & 32.76 & 32.09 & 31.68 & 31.27 \\
4.0444 & 47.64 & 36.83 & 31.89 & 31.16 & 30.42 & 30.23 & 30.11 & 29.59 & 28.45 \\
3.1576 & 39.10 & 30.20 & 29.54 & 29.30 & 29.06 & 28.94 & 28.76 & 27.76 & 26.83 \\
2.5758 & 28.93 & 28.73 & 28.30 & 28.20 & 28.08 & 27.96 & 27.63 & 26.27 & 25.53 \\
2.1589 & 26.85 & 27.62 & 27.53 & 27.46 & 27.36 & 27.11 & 26.57 & 25.28 & 24.50 \\
1.8422 & 26.07 & 27.07 & 26.98 & 26.90 & 26.78 & 26.42 & 25.70 & 24.48 & 23.64 \\
1.5915 & 25.40 & 26.61 & 26.52 & 26.43 & 26.21 & 25.80 & 24.94 & 23.77 & 22.90 \\
1.3868 & 24.66 & 25.98 & 25.92 & 25.78 & 25.49 & 24.97 & 24.15 & 23.07 & 22.17 \\
1.2156 & 24.43 & 25.76 & 25.67 & 25.49 & 25.18 & 24.50 & 23.77 & 22.75 & 21.85 \\
1.0696 & 23.79 & 25.16 & 25.02 & 24.82 & 24.51 & 23.72 & 23.07 & 22.05 & 21.14 \\
0.9432 & 23.42 & 24.85 & 24.61 & 24.38 & 24.03 & 23.12 & 22.58 & 21.59 & 20.77 \\
0.8322 & 23.16 & 24.57 & 24.24 & 23.96 & 23.46 & 22.61 & 22.08 & 20.98 & 20.12 \\
0.7336 & 22.83 & 24.19 & 23.81 & 23.46 & 22.86 & 22.16 & 21.67 & 20.61 & 19.80 \\
0.6453 & 22.34 & 23.62 & 23.19 & 22.76 & 22.10 & 21.49 & 20.98 & 19.91 & 19.15 \\
0.5655 & 22.07 & 23.24 & 22.74 & 22.27 & 21.59 & 21.06 & 20.55 & 19.49 & 18.74 \\
0.4928 & 21.75 & 22.83 & 22.24 & 21.75 & 21.11 & 20.61 & 20.08 & 19.04 & 18.28 \\
0.4263 & 21.50 & 22.47 & 21.79 & 21.30 & 20.72 & 20.22 & 19.67 & 18.64 & 17.84 \\
0.3650 & 20.88 & 21.82 & 21.08 & 20.63 & 20.09 & 19.61 & 19.09 & 18.11 & 17.30 \\
0.3082 & 20.47 & 21.38 & 20.55 & 20.13 & 19.66 & 19.17 & 18.64 & 17.68 & 16.84 \\
0.2554 & 19.99 & 20.89 & 19.97 & 19.58 & 19.14 & 18.64 & 18.11 & 17.17 & 16.37 \\
0.2061 & 19.50 & 20.32 & 19.38 & 19.03 & 18.60 & 18.11 & 17.58 & 16.64 & 15.91 \\
0.1599 & 18.65 & 19.38 & 18.49 & 18.16 & 17.75 & 17.28 & 16.77 & 15.86 & 15.20 \\
0.1165 & 17.85 & 18.43 & 17.64 & 17.33 & 16.92 & 16.45 & 15.94 & 15.03 & 14.44 \\
0.0756 & 16.93 & 17.37 & 16.66 & 16.35 & 15.94 & 15.46 & 14.95 & 14.04 & 13.49 \\
0.0369 & 15.33 & 15.66 & 15.01 & 14.72 & 14.31 & 13.81 & 13.33 & 12.41 & 11.93 \\
0.0000 & 4.23 & 4.47 & 3.88 & 3.60 & 3.20 & 2.69 & 2.22 & 1.31 & 0.90 \\
\hline
\end{tabular}


Table A.7. Apparent integrated SDSS magnitudes of the Kroupa model.

\begin{tabular}{rrrrrrrr}
\hline \hline$t[\mathrm{Gyr}]$ & $m_{\text {Bol }}$ & $m_{V_{j}}$ & $m_{u}$ & $m_{g}$ & $m_{r}$ & $m_{i}$ & $m_{z}$ \\
\hline 0.5 & 39.713 & 161.914 & 162.834 & 162.252 & 161.663 & 161.257 & 52.725 \\
1.0 & 33.463 & 39.350 & 59.066 & 56.723 & 36.533 & 34.342 & 32.664 \\
1.5 & 30.902 & 31.608 & 48.202 & 32.899 & 31.100 & 30.697 & 30.752 \\
2.0 & 29.206 & 29.453 & 34.143 & 29.948 & 29.375 & 29.425 & 29.524 \\
2.5 & 27.931 & 28.400 & 29.396 & 28.517 & 28.417 & 28.521 & 28.542 \\
3.0 & 26.905 & 27.635 & 28.073 & 27.635 & 27.681 & 27.761 & 27.638 \\
3.5 & 26.070 & 27.051 & 27.189 & 27.019 & 27.122 & 27.068 & 26.925 \\
4.0 & 25.406 & 26.659 & 26.604 & 26.604 & 26.674 & 26.499 & 26.301 \\
4.5 & 24.805 & 26.265 & 26.207 & 26.214 & 26.183 & 25.967 & 25.581 \\
5.0 & 24.177 & 25.705 & 25.682 & 25.723 & 25.567 & 25.377 & 24.708 \\
5.5 & 23.493 & 25.002 & 25.018 & 25.100 & 24.872 & 24.582 & 23.936 \\
6.0 & 22.906 & 24.446 & 24.553 & 24.602 & 24.318 & 23.810 & 23.343 \\
6.5 & 22.274 & 23.846 & 24.136 & 24.079 & 23.700 & 22.960 & 22.632 \\
7.0 & 22.016 & 23.639 & 23.986 & 23.857 & 23.432 & 22.697 & 22.474 \\
7.5 & 21.584 & 23.215 & 23.670 & 23.440 & 22.856 & 22.260 & 22.037 \\
8.0 & 21.177 & 22.754 & 23.359 & 23.031 & 22.279 & 21.801 & 21.571 \\
8.5 & 20.399 & 21.919 & 22.669 & 22.298 & 21.407 & 21.015 & 20.754 \\
9.0 & 20.074 & 21.521 & 22.521 & 22.055 & 21.037 & 20.700 & 20.409 \\
9.5 & 19.601 & 20.937 & 22.071 & 21.544 & 20.555 & 20.223 & 19.946 \\
10.0 & 19.139 & 20.381 & 21.640 & 21.010 & 20.085 & 19.736 & 19.472 \\
10.5 & 18.376 & 19.466 & 20.693 & 20.040 & 19.233 & 18.926 & 18.702 \\
11.0 & 17.796 & 18.847 & 20.151 & 19.391 & 18.635 & 18.321 & 18.087 \\
11.5 & 17.275 & 18.328 & 19.749 & 18.837 & 18.107 & 17.771 & 17.494 \\
12.0 & 16.448 & 17.490 & 18.954 & 17.946 & 17.258 & 16.939 & 16.625 \\
12.5 & 15.301 & 16.269 & 17.713 & 16.657 & 16.034 & 15.746 & 15.457 \\
13.0 & 13.690 & 14.640 & 16.146 & 15.008 & 14.389 & 14.101 & 13.791 \\
13.5 & 2.656 & 3.595 & 5.120 & 3.968 & 3.348 & 3.042 & 2.708 \\
\hline
\end{tabular}

Table A.8. Apparent integrated HST magnitudes of the Kroupa model.

\begin{tabular}{rrrrrrrrrr}
\hline \hline Redshift & $m_{U 336}$ & $m_{B 439}$ & $m_{V 555}$ & $m_{V 606}$ & $m_{R 675}$ & $m_{I 814}$ & $m_{J 110}$ & $m_{H 160}$ & $m_{K 222}$ \\
\hline 9.5116 & 161.8 & 162.6 & 162 & 161.7 & 161.2 & 58.14 & 39.58 & 36.74 & 36.49 \\
5.6177 & 57.31 & 59.76 & 39.03 & 36.31 & 34.76 & 32.72 & 32.05 & 31.63 & 31.19 \\
4.0444 & 47.65 & 36.89 & 31.93 & 31.19 & 30.44 & 30.24 & 30.11 & 29.57 & 28.39 \\
3.1576 & 39.21 & 30.30 & 29.62 & 29.37 & 29.12 & 28.99 & 28.80 & 27.78 & 26.83 \\
2.5758 & 29.07 & 28.85 & 28.40 & 28.31 & 28.17 & 28.05 & 27.72 & 26.34 & 25.62 \\
2.1589 & 27.00 & 27.75 & 27.65 & 27.57 & 27.46 & 27.21 & 26.65 & 25.36 & 24.59 \\
1.8422 & 26.15 & 27.15 & 27.05 & 26.97 & 26.85 & 26.50 & 25.79 & 24.58 & 23.74 \\
1.5915 & 25.53 & 26.73 & 26.64 & 26.54 & 26.32 & 25.92 & 25.06 & 23.89 & 23.01 \\
1.3868 & 24.99 & 26.30 & 26.25 & 26.10 & 25.79 & 25.25 & 24.41 & 23.29 & 22.31 \\
1.2156 & 24.49 & 25.81 & 25.72 & 25.52 & 25.19 & 24.51 & 23.75 & 22.66 & 21.66 \\
1.0696 & 23.85 & 25.22 & 25.05 & 24.83 & 24.51 & 23.70 & 23.05 & 22.00 & 21.02 \\
0.9432 & 23.35 & 24.78 & 24.51 & 24.28 & 23.93 & 23.02 & 22.46 & 21.39 & 20.44 \\
0.8322 & 22.91 & 24.32 & 23.92 & 23.63 & 23.13 & 22.27 & 21.74 & 20.64 & 19.74 \\
0.7336 & 22.76 & 24.10 & 23.70 & 23.36 & 22.77 & 22.08 & 21.56 & 20.42 & 19.50 \\
0.6453 & 22.45 & 23.69 & 23.27 & 22.86 & 22.21 & 21.64 & 21.11 & 19.94 & 19.05 \\
0.5655 & 22.14 & 23.28 & 22.80 & 22.35 & 21.70 & 21.18 & 20.65 & 19.49 & 18.66 \\
0.4928 & 21.48 & 22.55 & 21.99 & 21.51 & 20.89 & 20.38 & 19.83 & 18.66 & 17.82 \\
0.4263 & 21.40 & 22.32 & 21.65 & 21.16 & 20.58 & 20.04 & 19.45 & 18.26 & 17.38 \\
0.3650 & 20.97 & 21.85 & 21.11 & 20.66 & 20.11 & 19.57 & 18.95 & 17.77 & 16.87 \\
0.3082 & 20.55 & 21.42 & 20.58 & 20.15 & 19.65 & 19.10 & 18.46 & 17.30 & 16.37 \\
0.2554 & 19.60 & 20.54 & 19.65 & 19.26 & 18.82 & 18.30 & 17.72 & 16.65 & 15.79 \\
0.2061 & 19.07 & 19.93 & 19.00 & 18.64 & 18.20 & 17.70 & 17.10 & 16.05 & 15.26 \\
0.1599 & 18.69 & 19.39 & 18.46 & 18.11 & 17.65 & 17.13 & 16.50 & 15.44 & 14.72 \\
0.1165 & 17.89 & 18.45 & 17.61 & 17.26 & 16.81 & 16.27 & 15.63 & 14.58 & 13.91 \\
0.0756 & 16.63 & 17.10 & 16.37 & 16.04 & 15.61 & 15.10 & 14.49 & 13.48 & 12.90 \\
0.0369 & 15.08 & 15.40 & 14.73 & 14.40 & 13.97 & 13.43 & 12.84 & 11.83 & 11.28 \\
0.0000 & 4.11 & 4.32 & 3.68 & 3.37 & 2.94 & 2.36 & 1.77 & 0.75 & 0.26 \\
\hline
\end{tabular}


P. Westera et al.: Initial mass function effects on the colour evolution of disk galaxies, Online Material $p 6$

Appendix B: Mass-to-light ratios

Table B.1. Stellar mass-to-light ratios of the Salpeter model.

\begin{tabular}{|c|c|c|c|c|c|c|c|c|c|c|}
\hline$t[\mathrm{Gyr}]$ & $\overline{\bar{M} / L_{\mathrm{Bol}}}$ & $\overline{\bar{M} / L_{V_{j}}}$ & $\overline{\overline{M / L_{u}}}$ & $\overline{\bar{M} / L_{g}}$ & $\overline{\overline{M / L_{r}}}$ & $\overline{\overline{M / L_{i}}}$ & $\overline{\overline{M / L_{z}}}$ & 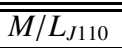 & $\overline{\bar{M} / L_{H 160}}$ & $\overline{M / L_{K 222}}$ \\
\hline 0.5 & 0.0075 & 0.1145 & 0.0137 & 0.0667 & 0.1568 & 0.2202 & 0.2757 & 0.4439 & 0.2919 & 0.5347 \\
\hline 1.0 & 0.0367 & 0.1787 & 0.0435 & 0.1253 & 0.2134 & 0.2503 & 0.2719 & 0.4022 & 0.1886 & 0.3014 \\
\hline 1.5 & 0.0720 & 0.2502 & 0.0759 & 0.1869 & 0.2881 & 0.3218 & 0.3326 & 0.4742 & 0.2012 & 0.309 \\
\hline 2.0 & 0.1098 & 0.322 & 1107 & 0.2511 & 0.3623 & 0.391 & 0.3896 & 0.5398 & 0.2143 & 0.3208 \\
\hline 2.5 & 0.1534 & 0.4061 & 0.1533 & 0.3274 & 0.4482 & 0.4689 & 4507 & 0.6079 & 0.2261 & 0.329 \\
\hline 3.0 & 0.2108 & 0.5262 & 0.2136 & 0.4357 & 0.5711 & 0.5807 & 0.5399 & 0.7079 & 63 & 0.34 \\
\hline 3.5 & 0.2838 & 0.6727 & 0.295 & 0.5715 & 0.7188 & 0.7148 & 83 & 21 & & 0.3793 \\
\hline 4.0 & 3602 & 0.8154 & 3824 & 0.7088 & 0.8593 & 0.839 & 0.7491 & .9483 & 35 & 0.4136 \\
\hline 4.5 & 4015 & 0.8998 & 0.4271 & 0.7844 & 0.9483 & 0.9292 & .832 & 1.05 & 03 & 0.4407 \\
\hline 5.0 & 5645 & 1.296 & 0.623 & 7 & & 25 & 3 & 67 & & 0.5441 \\
\hline 5.5 & 4 & 1.301 & 6267 & & & 38 & 11 & 57 & 98 & 0.5227 \\
\hline 6.0 & 1 & 1. & 3 & & & 4 & 1.323 & 5 & 88 & 268 \\
\hline 6.5 & 6 & 1.6 & 0.87 & 6 & 1. & 1. & 1.2 & 52 & 05 & 0.5906 \\
\hline 7.0 & 0.84 & 1.871 & 1.0 & 3 & 1. & 98 & 1.55 & 1.892 & 26 & 0.6805 \\
\hline 7.5 & 0.7877 & 1.669 & 0.9647 & 1.562 & 1.68 & 1.563 & 1.3 & 1.65 & 12 & 0.6692 \\
\hline 8.0 & 0.8925 & 1.865 & 1.109 & 1.768 & 1.863 & 1.718 & 1.466 & 1.802 & .5488 & 0.7315 \\
\hline 8.5 & 0.9728 & 2.021 & 1.245 & 1.934 & 2.008 & 1.84 & 1.559 & 1.904 & 0.5707 & 0.7559 \\
\hline 9.0 & 1.121 & 2.343 & 1.489 & 2.273 & 2.304 & 2.082 & 1.74 & 2.111 & 0.6248 & 0.8199 \\
\hline 9.5 & 8 & 2.25 & 1.417 & 2.175 & 2.221 & 2.026 & 1.707 & 2.085 & 67 & 0.8307 \\
\hline 10.0 & 1.219 & 2.56 & 1.629 & 2.488 & 2.516 & 2.274 & 1.901 & 2.309 & 0.6847 & 0.8951 \\
\hline & 1.327 & 2.809 & 1.828 & 2.757 & 2.742 & 2.457 & 2.038 & 2.466 & 79 & 0.949 \\
\hline 11 & 1.513 & 3.212 & 2.14 & 3.182 & 3.118 & 2.766 & 2.276 & 2.741 & 0.7978 & 1.022 \\
\hline & 1.402 & 2.945 & 1.98 & 2.916 & 2.8 & 2.561 & 2.1 & 2.56 & 0.7557 & 0.9879 \\
\hline & 1.476 & 3.093 & 2.138 & 3.093 & 2.9 & 2.652 & 2.179 & 2.625 & 0.7718 & 1.012 \\
\hline & & & 2.6 & & & 2.999 & & 2.912 & 53 & 1.093 \\
\hline & 1.902 & 3.953 & 2.982 & 4.063 & 3.761 & 3.279 & 2.6 & 3.177 & 81 & 1.173 \\
\hline 13.5 & 2.001 & 4.166 & 3.213 & 4.326 & 3.938 & 3.411 & 2.747 & 3.282 & 0.9491 & $1.21 \xi$ \\
\hline
\end{tabular}

Table B.2. Stellar mass-to-light ratios of the Kroupa model.

\begin{tabular}{|c|c|c|c|c|c|c|c|c|c|c|}
\hline$\overline{t \overline{\mathrm{G}} \mathrm{Gyr}]}$ & $\overline{\bar{c} M / L_{\mathrm{Bol}}}$ & $\overline{M / L_{V_{j}}}$ & $\overline{\overline{M / L_{u}}}$ & $\overline{\bar{M} M / L_{g}}$ & $\overline{\bar{M} / L_{r}}$ & $\overline{\bar{M} / L_{i}}$ & $\overline{\bar{M} / L_{z}}$ & $\overline{M / L_{J 110}}$ & $\overline{M / L_{H 160}}$ & $\overline{M / L_{K 222}}$ \\
\hline 0.5 & 0.0056 & 0.0643 & $\overline{0095}$ & 0.0397 & 0.0876 & 0.1255 & 0.1586 & 0.2544 & 0.1664 & 0.3011 \\
\hline 1.0 & 0.0288 & 0.1279 & 0.0330 & 0.0905 & 0.1563 & 0.1876 & 0.2012 & 0.2895 & 0.13 & 0.2078 \\
\hline 1.5 & 0.0568 & 0.1873 & 0.0586 & 0.1401 & 0.2198 & 0.2487 & 0.2533 & 16 & 428 & 0.2188 \\
\hline 2.0 & 0.0878 & 0.2487 & 0877 & 0.1936 & 0.2837 & 0.3079 & 0.302 & 078 & 45 & 0.229 \\
\hline 2.5 & 0.1242 & 0.3261 & 1248 & 0.2624 & 0.3635 & 0.38 & 595 & 0.4722 & 671 & 406 \\
\hline 3.0 & 0.168 & 0.4162 & 1706 & 0.3436 & 0.4551 & 4618 & 4243 & & 1808 & 524 \\
\hline 3.5 & 0.225 & 0.5502 & 0.24 & 19 & 0.5907 & 0.5842 & 11 & & 029 & 727 \\
\hline 4.0 & 0.2985 & 0.6991 & 3282 & 0.6 & 0.7388 & 0.7154 & & & 292 & 996 \\
\hline 4.5 & 3742 & 0.8661 & 4285 & 0.7648 & 0.9011 & 55 & & & 56 & 266 \\
\hline 5.0 & 8 & 0.9892 & 7 & & 22 & 0.9 & & & 73 & 491 \\
\hline 5. & 0.4385 & 1. & 5 & 0.9 & 3 & 8 & 13 & 23 & 86 & 614 \\
\hline 6.0 & 0.4868 & 1. & 4 & 5 & 5 & 1.115 & 84 & 1 & 46 & 747 \\
\hline 6.5 & 0.5041 & 1.119 & 0.6056 & 3 & 1.137 & 1.054 & 0.8777 & 36 & 66 & 761 \\
\hline 7.0 & 0.6922 & 1. & .8967 & 1.5 & 1. & 1.5 & 1.269 & 51 & 04 & 416 \\
\hline 7.5 & 0.7932 & 1.903 & 1.05 & 1.7 & 1.9 & 1.729 & 1.413 & 33 & 303 & 959 \\
\hline 8.0 & 0.9195 & 2.136 & 1.214 & 2.006 & 2.128 & 1.923 & 1.576 & 1.84 & 57 & 0.6008 \\
\hline 8.5 & 0.7509 & 1.727 & 1.041 & 1.653 & 1.697 & 1.526 & 1.234 & 1.416 & 69 & 0.4764 \\
\hline 9.0 & 0.9319 & 2.159 & 1.4 & 2.129 & 2.082 & 1.82 & 1.438 & 1.628 & 67 & 0.5333 \\
\hline 9.5 & 16 & 2.417 & 1.593 & 2.413 & 2.31 & 1.992 & 1.551 & 74 & & 646 \\
\hline 10.0 & 1.139 & 2.753 & 1.859 & 2.773 & 2.614 & 2.233 & 1.722 & 1.913 & 0.4981 & 0.6033 \\
\hline 10.5 & 0.9963 & 2.333 & 1.493 & 2.297 & 2.251 & 1.991 & 1.582 & 1.791 & 0.4844 & 0.5899 \\
\hline 11.0 & 1.078 & 2.516 & 1.676 & 2.506 & 2.409 & 2.118 & 1.67 & 1.88 & 0.5029 & 0.6079 \\
\hline 11.5 & 1.321 & 3.107 & 2.266 & 3.206 & 2.913 & 2.484 & 1.916 & 2.136 & 639 & 0.6761 \\
\hline & 1.377 & 3.295 & 2.492 & & 3.058 & 2.574 & 1.958 & 59 & 42 & 5833 \\
\hline 12.5 & 1.342 & 3.098 & 2.305 & 3.217 & 2.896 & 2.486 & 1.924 & 51 & 89 & 0.7083 \\
\hline & . & & 2.749 & 3.667 & 3.212 & 2.724 & 2.091 & 2.323 & 77 & 0.744 \\
\hline 3.5 & 1.683 & 3.997 & 3.34 & 4.315 & 3.656 & 3.052 & 2.307 & 2.534 & 0.6603 & 0.7859 \\
\hline
\end{tabular}


Table B.3. Stellar mass-to-light ratios of the absorptionless Salpeter model.

\begin{tabular}{|c|c|c|c|c|c|c|c|c|c|c|}
\hline Gyr] & $\overline{/ L_{\mathrm{Bol}}}$ & $\overline{\overline{I / L_{V_{j}}}}$ & $\overline{\bar{M} / L_{u}}$ & $\overline{\bar{M} / L_{g}}$ & $\overline{\bar{M} / L_{r}}$ & $\overline{\overline{M / L_{i}}}$ & $\overline{\bar{M} / L_{z}}$ & $\bar{M} M / L_{J 110}$ & $\overline{\bar{M} / L_{H 160}}$ & $M / L_{K 2}$ \\
\hline 0.5 & 0.0075 & 1145 & .0137 & 0.0667 & 0.1568 & 0.2202 & 0.2757 & 0.4439 & 0.2919 & 0.5347 \\
\hline 1.0 & 0339 & 0.1725 & 0413 & 0.1201 & 0.207 & 0.2443 & 0.2672 & 3966 & 74 & 30( \\
\hline 1.5 & 0594 & 57 & 658 & 55 & 0.2633 & 0.2998 & 0.3153 & 41 & 75 & 306 \\
\hline 2.0 & 0810 & 681 & 862 & 24 & 0.3081 & 0.3434 & .353 & 991 & 071 & 31 \\
\hline 2.5 & 0995 & 0.3038 & 0.1041 & 0.2343 & 0.346 & 0.3801 & 0.3839 & 0.5349 & 0.2133 & 3208 \\
\hline 3.0 & 1213 & 0.3461 & 0.125 & 0.2714 & 0.3908 & 0.4238 & 0.4214 & 0.5796 & 0.2238 & 0.3323 \\
\hline 3.5 & 0.1557 & 0.4113 & 0.159 & 0.3292 & 0.4586 & 0.4896 & 0.4788 & 0.6501 & 0.2433 & 0.3573 \\
\hline 4.0 & 0.1963 & 0.4918 & 0.2018 & 0.4023 & 0.5407 & 0.5667 & 0.5462 & 0.7314 & 0.2653 & 0.386 \\
\hline 4.5 & 0.2237 & 0.5563 & 0.2321 & 0.4606 & 0.6066 & 0.6277 & 0.595 & 0.7858 & 0.277 & 0.3986 \\
\hline 5.0 & 0.2941 & 0.68 & 0.3119 & 0.5777 & 0.7306 & 0.7435 & 0.6971 & 0.9122 & 0.3157 & 4513 \\
\hline 5.5 & 0.3115 & 7432 & 0.3372 & 0.6337 & 0.7949 & 0.8044 & 0.7494 & 977 & 35 & 0.4772 \\
\hline 6.0 & 3521 & .8217 & 0.3826 & 0.7065 & 0.874 & 0.8797 & 0.8142 & 59 & 607 & 123 \\
\hline 6.5 & 4124 & 9291 & 4521 & 0.8107 & 0.9792 & 0.9738 & 0.8914 & 1.15 & 66 & 0.5461 \\
\hline 7.0 & 4761 & 1.05 & 0.5317 & 0.9308 & 1.097 & 1.078 & 0.9774 & 253 & 0.4154 & 0.5846 \\
\hline 7.5 & 5405 & 1.18 & 0.6177 & 1.06 & 1.222 & 1.189 & 1.069 & 361 & 0.4467 & 0.6257 \\
\hline 8.0 & 5891 & 1.288 & 0.682 & 1.164 & 1.327 & 1.284 & 1.146 & 54 & 0.4736 & 0.6604 \\
\hline 8.5 & 6626 & 1.428 & 0.7857 & 1.307 & 1.46 & 1.4 & 1.239 & 66 & 0.5059 & 0.7035 \\
\hline 9.0 & 0.7341 & 1.564 & 0.885 & 1.446 & 1.59 & 1.511 & 1.33 & 572 & 0.5367 & 0.7429 \\
\hline 9.5 & 0.781 & 1.682 & 0.9564 & 1.563 & 1.702 & 1.612 & 1.409 & 1.768 & 0.5642 & 0.7788 \\
\hline 10.0 & 0.8262 & 1.789 & 1.021 & 1.668 & 1.808 & 1.704 & 1.483 & 1.855 & 0.5898 & 0.8119 \\
\hline 10.5 & 0.8997 & 1.929 & 1.134 & 1.815 & 1.938 & 1.815 & 1.571 & 1.961 & 0.6201 & 0.8521 \\
\hline 11.0 & 0.9628 & 2.053 & 1.226 & 1.942 & 2.053 & 1.914 & 1.649 & 2.051 & 0.6455 & 0.8837 \\
\hline 11.5 & 1.045 & 2.208 & 1.357 & 2.11 & 2.195 & 2.032 & 1.739 & 2.157 & 0.676 & 0.9228 \\
\hline 12.0 & 1.121 & 2.368 & 1.483 & 2.28 & 2.339 & 2.153 & 1.831 & 2.263 & 0.7046 & 0.9592 \\
\hline 12.5 & 1.217 & 2.553 & 1.65 & 2.488 & 2.509 & 2.292 & 1.935 & 2.382 & 0.7376 & 0.9996 \\
\hline 13.0 & 1.374 & 2.803 & 1.938 & 2.78 & 2.727 & 2.466 & 2.065 & 2.531 & 0.7785 & 1.051 \\
\hline 13.5 & 1.438 & 2.996 & 2.065 & 2.987 & 2.901 & 2.607 & 2.17 & 2.653 & 0.8115 & 1.094 \\
\hline
\end{tabular}

Table B.4. Stellar mass-to-light ratios of the absorptionless Kroupa model.

\begin{tabular}{|c|c|c|c|c|c|c|c|c|c|c|}
\hline$t[\mathrm{Gyr}]$ & $M / L_{\mathrm{Bol}}$ & $M / L_{V_{j}}$ & $M / L_{u}$ & $M / L_{g}$ & $M / L_{r}$ & $M / L_{i}$ & $M / L_{z}$ & $M / L_{J 110}$ & $M / L_{H 160}$ & $M / L_{K 222}$ \\
\hline 0.5 & 0.0056 & 0.0643 & 0.0095 & 0.0397 & 0.0876 & 0.1255 & 0.1586 & 0.2544 & 0.1664 & 0.3011 \\
\hline 1.0 & 0.0264 & 0.1229 & 311 & 0.0863 & 0.151 & 1825 & 0.1972 & 0.285 & & 1 \\
\hline 1.5 & 0.0461 & 0.167 & 500 & 25 & 0.1988 & 2295 & 0.2386 & 52 & & 166 \\
\hline 2.0 & 0.0638 & 0.2034 & 58 & & 0.2373 & 2665 & 07 & 74 & & 2253 \\
\hline 2.5 & 0782 & 2356 & 814 & 07 & 0.271 & 2985 & 82 & 68 & & 326 \\
\hline 3.0 & 0.0934 & 77 & 0965 & 0.2088 & 0.3043 & 33 & 52 & 82 & 63 & 391 \\
\hline 3.5 & 0.114 & 3 & 76 & +6 & 0.3478 & 0.371 & 05 & 01 & 37 & 252 \\
\hline 4.0 & 0.1438 & 582 & 0.1484 & 0.2979 & 0.4071 & 0.4271 & 0.4086 & 77 & 92 & $2 / 1$ \\
\hline 4.5 & 13 & 55 & 81 & 5 & 0.4699 & 0.4852 & 75 & 53 & 47 & 0.2891 \\
\hline 5.0 & 0.1976 & 0.4867 & 0.209 & 0.4052 & 0.5269 & 0.5396 & 0.5022 & 47 & 86 & 0.306 \\
\hline 5.5 & 0.2017 & 0. & 0.2173 & 0.4293 & 0.5576 & 0.5696 & 0.5272 & 0.6747 & 59 & 0.3136 \\
\hline 6.0 & 0.2142 & 0.5447 & 0.2296 & 0.4539 & 0.5879 & 0.5988 & 0.5492 & 0.6978 & 308 & 0.3178 \\
\hline 6.5 & 0.2504 & 0.6044 & 0.2683 & 0.5097 & 0.6471 & 0.6531 & 0.5918 & 0.7429 & 15 & 0.3279 \\
\hline 7.0 & 0.3069 & 0.7175 & 0.3422 & 0.6221 & 0.7548 & 0.7486 & 0.6715 & 53 & & 589 \\
\hline 7.5 & 0.3425 & 0.802 & 0.3903 & 0.7037 & 0.8368 & 0.823 & 0.7 & & & 832 \\
\hline 8.0 & 0.3777 & 8829 & 4368 & 0.7804 & 0.9152 & 0.8944 & 0.7891 & 99 & 45 & 0.4062 \\
\hline 8.5 & 0.4192 & 0.9718 & 0.4934 & 0.8677 & 1.001 & 0.97 & 48 & 35 & & 0.4269 \\
\hline 9.0 & 0.4714 & 1.082 & 5693 & 0.9794 & 1.104 & 1.06 & 83 & 13 & & 0.4527 \\
\hline 9.5 & 0.5102 & $1.1 / 7$ & 0. & 74 & 1.195 & 1.14 & 0.9802 & 82 & 05 & 0.4739 \\
\hline 10.0 & 5 & 1.2 & 6 & 36 & 1.297 & 23 & 1.05 & 58 & 07 & 986 \\
\hline & 7 & 1.393 & 59 & 94 & 1.397 & 1.316 & 15 & 29 & 92 & 204 \\
\hline & 0.6 & 1. & 0.8659 & 1.4 & 1.505 & 1.409 & 35 & 1.404 & 19 & 438 \\
\hline .5 & 0.7284 & 1.653 & 0.979 & 1.572 & 1.635 & 1.518 & 1.268 & 1.494 & 26 & 0.5717 \\
\hline 12.0 & 0.7717 & 1.7 & 1.05 & 1.6 & 1.739 & 1.608 & 1.335 & 1.565 & 07 & 0.5935 \\
\hline 12.5 & 9 & 1.908 & 1.172 & 1.839 & 1.868 & 1.716 & 1.415 & 1.65 & 0.4824 & 0.6186 \\
\hline & 9094 & 2.064 & 1.307 & 2.012 & 2.006 & 1.831 & 1.498 & 1.739 & 0.5053 & 0.6456 \\
\hline 13.5 & 0.9867 & 2.238 & 1.465 & 2.207 & 2.161 & 1.956 & 1.59 & 1.836 & 0.5304 & 0.6751 \\
\hline
\end{tabular}


P. Westera et al.: Initial mass function effects on the colour evolution of disk galaxies, Online Material $p 8$

\section{Appendix C: Correction tables}

Table C.1. Absorption corrections $m_{\mathrm{X}, \text { includingabs. }}-m_{\mathrm{X}, \text { abs.less }}$ for the Kroupa model in the SDSS system.

\begin{tabular}{rrrrrrrr}
\hline \hline Redshift & BOL & $V_{j}$ & $u$ & $g$ & $r$ & $i$ & $z$ \\
\hline 9.5116 & 0 & 0 & 0 & 0 & 0 & 0 & 0 \\
5.6177 & 0.081 & 0.152 & 0.258 & 0.151 & 0.151 & 0.124 & 0.105 \\
4.0444 & 0.210 & 0.339 & 0.498 & 0.378 & 0.294 & 0.259 & 0.254 \\
3.1576 & 0.331 & 0.447 & 0.631 & 0.506 & 0.420 & 0.412 & 0.458 \\
2.5758 & 0.488 & 0.627 & 0.827 & 0.669 & 0.620 & 0.677 & 0.607 \\
2.1589 & 0.625 & 0.820 & 0.974 & 0.840 & 0.848 & 0.849 & 0.711 \\
1.8422 & 0.727 & 0.992 & 1.037 & 0.966 & 1.045 & 0.912 & 0.824 \\
1.5915 & 0.783 & 1.157 & 1.123 & 1.094 & 1.117 & 0.959 & 0.876 \\
1.3868 & 0.828 & 1.247 & 1.187 & 1.224 & 1.121 & 0.998 & 0.911 \\
1.2156 & 0.820 & 1.163 & 1.166 & 1.233 & 1.060 & 0.965 & 0.893 \\
1.0696 & 0.837 & 1.105 & 1.179 & 1.234 & 1.027 & 0.934 & 0.878 \\
0.9432 & 0.886 & 1.147 & 1.278 & 1.291 & 1.081 & 0.980 & 0.917 \\
0.8322 & 0.757 & 0.991 & 1.241 & 1.147 & 0.918 & 0.818 & 0.736 \\
0.7336 & 0.880 & 1.094 & 1.339 & 1.183 & 1.044 & 0.999 & 0.938 \\
0.6453 & 0.908 & 1.101 & 1.402 & 1.178 & 1.052 & 1.016 & 0.941 \\
0.5655 & 0.962 & 1.115 & 1.446 & 1.195 & 1.070 & 1.022 & 0.945 \\
0.4928 & 0.631 & 0.794 & 1.089 & 0.873 & 0.753 & 0.668 & 0.585 \\
0.4263 & 0.738 & 0.932 & 1.272 & 1.027 & 0.886 & 0.776 & 0.681 \\
0.3650 & 0.746 & 0.952 & 1.243 & 1.032 & 0.905 & 0.781 & 0.683 \\
0.3082 & 0.765 & 0.977 & 1.238 & 1.045 & 0.924 & 0.801 & 0.704 \\
0.2554 & 0.534 & 0.649 & 0.801 & 0.692 & 0.607 & 0.530 & 0.469 \\
0.2061 & 0.524 & 0.634 & 0.801 & 0.684 & 0.584 & 0.509 & 0.445 \\
0.1599 & 0.645 & 0.773 & 1.008 & 0.845 & 0.702 & 0.606 & 0.520 \\
0.1165 & 0.629 & 0.754 & 1.020 & 0.842 & 0.673 & 0.570 & 0.474 \\
0.0756 & 0.508 & 0.564 & 0.774 & 0.640 & 0.506 & 0.431 & 0.360 \\
0.0369 & 0.543 & 0.584 & 0.831 & 0.672 & 0.526 & 0.447 & 0.374 \\
0.0000 & 0.580 & 0.630 & 0.895 & 0.728 & 0.571 & 0.483 & 0.404 \\
\hline & & & & & & &
\end{tabular}

Table C.2. Absorption corrections $m_{\mathrm{X} \text {,includingabs. }}-m_{\mathrm{X}, \text { abs.less }}$ for the Kroupa model in the HST system.

\begin{tabular}{rrrrrrrrrr}
\hline \hline Redshift & $U 336$ & $B 439$ & $V 555$ & $V 606$ & $R 675$ & $I 814$ & $J 110$ & $H 160$ & $K 222$ \\
\hline 9.5116 & 0 & 0 & 0 & 0 & 0 & 0 & 0 & 0 & 0 \\
5.6177 & 0.267 & 0.199 & 0.151 & 0.143 & 0.139 & 0.107 & 0.100 & 0.100 & 0.065 \\
4.0444 & 0.546 & 0.422 & 0.340 & 0.307 & 0.276 & 0.255 & 0.261 & 0.191 & 0.154 \\
3.1576 & 0.627 & 0.553 & 0.459 & 0.435 & 0.413 & 0.436 & 0.396 & 0.283 & 0.222 \\
2.5758 & 0.869 & 0.705 & 0.641 & 0.629 & 0.634 & 0.649 & 0.536 & 0.419 & 0.317 \\
2.1589 & 1.024 & 0.862 & 0.824 & 0.841 & 0.887 & 0.770 & 0.641 & 0.516 & 0.391 \\
1.8422 & 1.069 & 0.967 & 0.986 & 1.004 & 1.004 & 0.861 & 0.757 & 0.617 & 0.472 \\
1.5915 & 1.157 & 1.079 & 1.125 & 1.114 & 1.028 & 0.912 & 0.805 & 0.651 & 0.484 \\
1.3868 & 1.215 & 1.177 & 1.230 & 1.177 & 1.053 & 0.944 & 0.842 & 0.674 & 0.495 \\
1.2156 & 1.169 & 1.201 & 1.199 & 1.119 & 1.012 & 0.918 & 0.824 & 0.651 & 0.473 \\
1.0696 & 1.180 & 1.266 & 1.157 & 1.071 & 0.985 & 0.897 & 0.802 & 0.619 & 0.442 \\
0.9432 & 1.270 & 1.375 & 1.188 & 1.115 & 1.032 & 0.942 & 0.830 & 0.628 & 0.453 \\
0.8322 & 1.204 & 1.258 & 1.029 & 0.950 & 0.863 & 0.771 & 0.646 & 0.446 & 0.305 \\
0.7336 & 1.289 & 1.249 & 1.112 & 1.063 & 1.015 & 0.966 & 0.868 & 0.683 & 0.506 \\
0.6453 & 1.373 & 1.230 & 1.117 & 1.072 & 1.034 & 0.975 & 0.870 & 0.685 & 0.527 \\
0.5655 & 1.469 & 1.241 & 1.133 & 1.088 & 1.052 & 0.977 & 0.870 & 0.712 & 0.582 \\
0.4928 & 1.142 & 0.918 & 0.811 & 0.763 & 0.717 & 0.619 & 0.508 & 0.339 & 0.219 \\
0.4263 & 1.367 & 1.081 & 0.951 & 0.898 & 0.840 & 0.720 & 0.584 & 0.391 & 0.268 \\
0.3650 & 1.329 & 1.087 & 0.965 & 0.915 & 0.850 & 0.724 & 0.577 & 0.379 & 0.257 \\
0.3082 & 1.309 & 1.099 & 0.987 & 0.937 & 0.866 & 0.745 & 0.593 & 0.393 & 0.272 \\
0.2554 & 0.839 & 0.722 & 0.656 & 0.620 & 0.568 & 0.495 & 0.400 & 0.271 & 0.183 \\
0.2061 & 0.836 & 0.716 & 0.643 & 0.602 & 0.546 & 0.473 & 0.377 & 0.251 & 0.161 \\
0.1599 & 1.057 & 0.887 & 0.788 & 0.730 & 0.654 & 0.556 & 0.442 & 0.306 & 0.214 \\
0.1165 & 1.072 & 0.891 & 0.774 & 0.706 & 0.624 & 0.514 & 0.392 & 0.249 & 0.171 \\
0.0756 & 0.814 & 0.683 & 0.584 & 0.532 & 0.470 & 0.389 & 0.308 & 0.211 & 0.157 \\
0.0369 & 0.880 & 0.725 & 0.606 & 0.552 & 0.489 & 0.404 & 0.325 & 0.225 & 0.160 \\
0.0000 & 0.945 & 0.785 & 0.655 & 0.596 & 0.531 & 0.436 & 0.350 & 0.238 & 0.165 \\
\hline & & & & & & & & &
\end{tabular}


P. Westera et al.: Initial mass function effects on the colour evolution of disk galaxies, Online Material $p 9$

Table C.3. Inclination corrections $m_{\mathrm{X}, \text { diagonal }}-m_{\mathrm{X} \text {,faceon }}$ for the Kroupa model in the SDSS system.

\begin{tabular}{rrrrrrrrr}
\hline \hline Redshift & BOL & $V_{j}$ & $u$ & $g$ & $r$ & $i$ & $z$ \\
\hline 9.5116 & 0 & 0 & 0 & 0 & 0 & 0 & 0 \\
5.6177 & 0.037 & 0.065 & 0.079 & 0.049 & 0.064 & 0.055 & 0.047 \\
4.0444 & 0.071 & 0.110 & 0.121 & 0.121 & 0.098 & 0.088 & 0.086 \\
3.1576 & 0.119 & 0.160 & 0.211 & 0.174 & 0.152 & 0.151 & 0.163 \\
2.5758 & 0.170 & 0.219 & 0.257 & 0.227 & 0.218 & 0.233 & 0.217 \\
2.1589 & 0.243 & 0.329 & 0.378 & 0.336 & 0.337 & 0.335 & 0.290 \\
1.8422 & 0.275 & 0.378 & 0.377 & 0.365 & 0.396 & 0.358 & 0.334 \\
1.5915 & 0.332 & 0.501 & 0.485 & 0.476 & 0.485 & 0.422 & 0.388 \\
1.3868 & 0.365 & 0.567 & 0.539 & 0.557 & 0.509 & 0.453 & 0.412 \\
1.2156 & 0.378 & 0.557 & 0.553 & 0.591 & 0.506 & 0.460 & 0.424 \\
1.0696 & 0.397 & 0.543 & 0.578 & 0.609 & 0.503 & 0.454 & 0.425 \\
0.9432 & 0.436 & 0.597 & 0.657 & 0.667 & 0.563 & 0.508 & 0.475 \\
0.8322 & 0.218 & 0.347 & 0.475 & 0.424 & 0.311 & 0.264 & 0.226 \\
0.7336 & 0.260 & 0.390 & 0.456 & 0.415 & 0.375 & 0.361 & 0.338 \\
0.6453 & 0.304 & 0.427 & 0.551 & 0.464 & 0.403 & 0.377 & 0.341 \\
0.5655 & 0.360 & 0.455 & 0.594 & 0.493 & 0.432 & 0.402 & 0.363 \\
0.4928 & 0.036 & 0.141 & 0.239 & 0.174 & 0.118 & 0.064 & 0.019 \\
0.4263 & 0.133 & 0.275 & 0.445 & 0.326 & 0.239 & 0.165 & 0.102 \\
0.3650 & 0.157 & 0.320 & 0.504 & 0.376 & 0.277 & 0.188 & 0.118 \\
0.3082 & 0.176 & 0.330 & 0.502 & 0.382 & 0.285 & 0.197 & 0.129 \\
0.2554 & -0.049 & 0.006 & 0.082 & 0.037 & -0.023 & -0.069 & -0.100 \\
0.2061 & -0.095 & -0.056 & 0.024 & -0.022 & -0.082 & -0.124 & -0.154 \\
0.1599 & 0.058 & 0.119 & 0.302 & 0.183 & 0.065 & -0.008 & -0.066 \\
0.1165 & 0.007 & 0.092 & 0.295 & 0.166 & 0.030 & -0.049 & -0.120 \\
0.0756 & -0.098 & -0.116 & 0.063 & -0.055 & -0.160 & -0.210 & -0.252 \\
0.0369 & -0.083 & -0.113 & 0.075 & -0.049 & -0.157 & -0.208 & -0.254 \\
0.0000 & -0.008 & -0.024 & 0.179 & 0.049 & -0.069 & -0.129 & -0.183 \\
\hline
\end{tabular}

Table C.4. Inclination corrections $m_{\mathrm{X}, \mathrm{diagonal}}-m_{\mathrm{X} \text {,faceon }}$ for the Kroupa model in the HST system.

\begin{tabular}{rrrrrrrrrr}
\hline \hline Redshift & $U 336$ & $B 439$ & $V 555$ & $V 606$ & $R 675$ & $I 814$ & $J 110$ & $H 160$ & $K 222$ \\
\hline 9.5116 & 0 & 0 & 0 & 0 & 0 & 0 & 0 & 0 & 0 \\
5.6177 & 0.081 & 0.062 & 0.065 & 0.061 & 0.060 & 0.048 & 0.045 & 0.045 & 0.030 \\
4.0444 & 0.128 & 0.137 & 0.110 & 0.101 & 0.093 & 0.087 & 0.088 & 0.067 & 0.054 \\
3.1576 & 0.206 & 0.184 & 0.163 & 0.157 & 0.151 & 0.158 & 0.145 & 0.111 & 0.090 \\
2.5758 & 0.267 & 0.234 & 0.222 & 0.220 & 0.222 & 0.226 & 0.198 & 0.167 & 0.134 \\
2.1589 & 0.392 & 0.345 & 0.330 & 0.334 & 0.348 & 0.310 & 0.262 & 0.213 & 0.170 \\
1.8422 & 0.379 & 0.363 & 0.374 & 0.382 & 0.384 & 0.344 & 0.313 & 0.269 & 0.218 \\
1.5915 & 0.498 & 0.468 & 0.489 & 0.484 & 0.450 & 0.403 & 0.357 & 0.298 & 0.235 \\
1.3868 & 0.554 & 0.536 & 0.559 & 0.534 & 0.479 & 0.428 & 0.383 & 0.317 & 0.250 \\
1.2156 & 0.553 & 0.574 & 0.574 & 0.535 & 0.484 & 0.437 & 0.393 & 0.318 & 0.245 \\
1.0696 & 0.576 & 0.626 & 0.569 & 0.525 & 0.481 & 0.435 & 0.390 & 0.310 & 0.236 \\
0.9432 & 0.652 & 0.710 & 0.616 & 0.579 & 0.537 & 0.488 & 0.432 & 0.333 & 0.243 \\
0.8322 & 0.457 & 0.481 & 0.366 & 0.326 & 0.285 & 0.242 & 0.182 & 0.079 & -0.002 \\
0.7336 & 0.443 & 0.433 & 0.396 & 0.381 & 0.366 & 0.349 & 0.309 & 0.216 & 0.097 \\
0.6453 & 0.542 & 0.488 & 0.436 & 0.412 & 0.391 & 0.357 & 0.303 & 0.198 & 0.113 \\
0.5655 & 0.603 & 0.516 & 0.463 & 0.440 & 0.420 & 0.379 & 0.324 & 0.238 & 0.190 \\
0.4928 & 0.248 & 0.195 & 0.148 & 0.121 & 0.090 & 0.038 & -0.018 & -0.114 & -0.143 \\
0.4263 & 0.482 & 0.357 & 0.284 & 0.247 & 0.205 & 0.128 & 0.042 & -0.081 & -0.099 \\
0.3650 & 0.546 & 0.411 & 0.328 & 0.288 & 0.237 & 0.147 & 0.042 & -0.106 & -0.136 \\
0.3082 & 0.539 & 0.417 & 0.337 & 0.299 & 0.245 & 0.158 & 0.049 & -0.083 & -0.085 \\
0.2554 & 0.095 & 0.049 & 0.012 & -0.012 & -0.045 & -0.088 & -0.143 & -0.197 & -0.154 \\
0.2061 & 0.037 & -0.006 & -0.048 & -0.072 & -0.103 & -0.141 & -0.194 & -0.225 & -0.162 \\
0.1599 & 0.336 & 0.222 & 0.135 & 0.086 & 0.027 & -0.042 & -0.117 & -0.148 & -0.083 \\
0.1165 & 0.331 & 0.210 & 0.110 & 0.055 & -0.010 & -0.091 & -0.188 & -0.254 & -0.189 \\
0.0756 & 0.093 & -0.011 & -0.100 & -0.140 & -0.185 & -0.234 & -0.284 & -0.272 & -0.169 \\
0.0369 & 0.108 & -0.007 & -0.099 & -0.138 & -0.182 & -0.235 & -0.276 & -0.231 & -0.126 \\
0.0000 & 0.212 & 0.093 & -0.006 & -0.049 & -0.096 & -0.161 & -0.203 & -0.161 & -0.079 \\
\hline
\end{tabular}


Table C.5. Inclination corrections $m_{\mathrm{X}, \text { edgeon }}-m_{\mathrm{X}, \text { faceon }}$ for the Kroupa model in the SDSS system.

\begin{tabular}{rrrrrrrr}
\hline \hline Redshift & BOL & $V_{j}$ & $u$ & $g$ & $r$ & $i$ & $z$ \\
\hline 9.5116 & 0 & 0 & 0 & 0 & 0 & 0 & 0 \\
5.6177 & 0.067 & 0.121 & 0.145 & 0.072 & 0.119 & 0.1 & 0.086 \\
4.0444 & 0.150 & 0.228 & 0.203 & 0.248 & 0.207 & 0.189 & 0.185 \\
3.1576 & 0.259 & 0.34 & 0.419 & 0.359 & 0.328 & 0.326 & 0.345 \\
2.5758 & 0.342 & 0.426 & 0.478 & 0.438 & 0.426 & 0.447 & 0.425 \\
2.1589 & 0.482 & 0.613 & 0.637 & 0.614 & 0.624 & 0.625 & 0.584 \\
1.8422 & 0.576 & 0.746 & 0.746 & 0.729 & 0.767 & 0.723 & 0.693 \\
1.5915 & 0.700 & 0.943 & 0.918 & 0.914 & 0.929 & 0.870 & 0.831 \\
1.3868 & 0.791 & 1.098 & 1.063 & 1.088 & 1.034 & 0.969 & 0.911 \\
1.2156 & 0.869 & 1.170 & 1.172 & 1.212 & 1.108 & 1.040 & 0.974 \\
1.0696 & 0.930 & 1.165 & 1.185 & 1.226 & 1.131 & 1.078 & 1.049 \\
0.9432 & 1.034 & 1.327 & 1.374 & 1.393 & 1.293 & 1.220 & 1.188 \\
0.8322 & 1.004 & 1.318 & 1.382 & 1.362 & 1.290 & 1.236 & 1.207 \\
0.7336 & 0.856 & 1.189 & 1.286 & 1.228 & 1.156 & 1.120 & 1.086 \\
0.6453 & 0.894 & 1.226 & 1.385 & 1.282 & 1.179 & 1.141 & 1.095 \\
0.5655 & 0.916 & 1.261 & 1.440 & 1.322 & 1.217 & 1.178 & 1.129 \\
0.4928 & 0.900 & 1.252 & 1.375 & 1.296 & 1.229 & 1.197 & 1.150 \\
0.4263 & 0.900 & 1.279 & 1.452 & 1.340 & 1.254 & 1.200 & 1.145 \\
0.3650 & 0.943 & 1.360 & 1.603 & 1.438 & 1.325 & 1.249 & 1.180 \\
0.3082 & 0.861 & 1.311 & 1.460 & 1.353 & 1.282 & 1.208 & 1.130 \\
0.2554 & 0.902 & 1.338 & 1.506 & 1.381 & 1.298 & 1.216 & 1.129 \\
0.2061 & 0.837 & 1.318 & 1.515 & 1.370 & 1.264 & 1.172 & 1.065 \\
0.1599 & 0.757 & 1.293 & 1.490 & 1.352 & 1.224 & 1.107 & 0.960 \\
0.1165 & 0.726 & 1.297 & 1.520 & 1.375 & 1.213 & 1.073 & 0.892 \\
0.0756 & 0.687 & 1.229 & 1.482 & 1.329 & 1.136 & 0.975 & 0.772 \\
0.0369 & 0.583 & 1.127 & 1.403 & 1.247 & 1.025 & 0.840 & 0.613 \\
0.0000 & 0.501 & 0.957 & 1.294 & 1.107 & 0.848 & 0.649 & 0.432 \\
\hline
\end{tabular}

Table C.6. Inclination corrections $m_{\mathrm{X}, \text { diagonal }}-m_{\mathrm{X}, \text { edgeon }}$ for the Kroupa model in the HST system.

\begin{tabular}{rrrrrrrrrr}
\hline \hline Redshift & $U 336$ & $B 439$ & $V 555$ & $V 606$ & $R 675$ & $I 814$ & $J 110$ & $H 160$ & $K 222$ \\
\hline 9.5116 & 0 & 0 & 0 & 0 & 0 & 0 & 0 & 0 & 0 \\
5.6177 & 0.150 & 0.105 & 0.121 & 0.114 & 0.111 & 0.088 & 0.083 & 0.083 & 0.055 \\
4.0444 & 0.217 & 0.289 & 0.229 & 0.213 & 0.198 & 0.186 & 0.187 & 0.145 & 0.119 \\
3.1576 & 0.407 & 0.372 & 0.344 & 0.335 & 0.326 & 0.336 & 0.317 & 0.257 & 0.214 \\
2.5758 & 0.493 & 0.447 & 0.431 & 0.428 & 0.432 & 0.438 & 0.400 & 0.356 & 0.303 \\
2.1589 & 0.645 & 0.622 & 0.611 & 0.618 & 0.636 & 0.602 & 0.551 & 0.492 & 0.426 \\
1.8422 & 0.745 & 0.724 & 0.741 & 0.751 & 0.752 & 0.705 & 0.661 & 0.601 & 0.525 \\
1.5915 & 0.930 & 0.903 & 0.930 & 0.927 & 0.896 & 0.849 & 0.791 & 0.716 & 0.619 \\
1.3868 & 1.084 & 1.065 & 1.089 & 1.062 & 1.000 & 0.934 & 0.873 & 0.788 & 0.682 \\
1.2156 & 1.172 & 1.195 & 1.189 & 1.141 & 1.076 & 0.996 & 0.935 & 0.839 & 0.731 \\
1.0696 & 1.175 & 1.242 & 1.189 & 1.150 & 1.112 & 1.058 & 1.015 & 0.917 & 0.796 \\
0.9432 & 1.365 & 1.432 & 1.344 & 1.308 & 1.262 & 1.200 & 1.141 & 1.018 & 0.855 \\
0.8322 & 1.366 & 1.394 & 1.328 & 1.298 & 1.258 & 1.219 & 1.157 & 1.015 & 0.815 \\
0.7336 & 1.269 & 1.255 & 1.197 & 1.166 & 1.131 & 1.102 & 1.039 & 0.875 & 0.611 \\
0.6453 & 1.376 & 1.314 & 1.238 & 1.196 & 1.156 & 1.116 & 1.042 & 0.858 & 0.587 \\
0.5655 & 1.449 & 1.353 & 1.274 & 1.233 & 1.199 & 1.150 & 1.065 & 0.863 & 0.567 \\
0.4928 & 1.394 & 1.315 & 1.262 & 1.239 & 1.220 & 1.170 & 1.078 & 0.848 & 0.538 \\
0.4263 & 1.484 & 1.371 & 1.292 & 1.262 & 1.233 & 1.168 & 1.057 & 0.796 & 0.485 \\
0.3650 & 1.647 & 1.487 & 1.376 & 1.335 & 1.292 & 1.210 & 1.070 & 0.755 & 0.413 \\
0.3082 & 1.490 & 1.389 & 1.317 & 1.289 & 1.249 & 1.164 & 0.992 & 0.637 & 0.331 \\
0.2554 & 1.541 & 1.421 & 1.345 & 1.310 & 1.259 & 1.167 & 0.990 & 0.649 & 0.361 \\
0.2061 & 1.558 & 1.410 & 1.326 & 1.283 & 1.220 & 1.111 & 0.896 & 0.526 & 0.274 \\
0.1599 & 1.528 & 1.387 & 1.304 & 1.249 & 1.172 & 1.022 & 0.747 & 0.359 & 0.165 \\
0.1165 & 1.561 & 1.412 & 1.314 & 1.244 & 1.152 & 0.967 & 0.658 & 0.252 & 0.080 \\
0.0756 & 1.524 & 1.375 & 1.253 & 1.172 & 1.066 & 0.856 & 0.552 & 0.201 & 0.080 \\
0.0369 & 1.448 & 1.302 & 1.157 & 1.063 & 0.946 & 0.705 & 0.400 & 0.098 & 0.022 \\
0.0000 & 1.343 & 1.184 & 0.995 & 0.889 & 0.762 & 0.518 & 0.265 & 0.050 & 0.013 \\
\hline & & & & & & & & &
\end{tabular}


P. Westera et al.: Initial mass function effects on the colour evolution of disk galaxies, Online Material p 11

\section{Appendix D: Other quantities}

Table D.1. Petrosion radii $[\mathrm{kpc}]$ for the Kroupa model.

\begin{tabular}{rrrrrrrr}
\hline \hline$t[\mathrm{Gyr}]$ & petroR90 BOL & petroR90 & petroR90 & petroR90 & petroR90 & petroR90 & petroR90 \\
\hline 0.5 & 16.69 & 16.72 & 16.71 & 16.72 & 16.73 & 16.62 & 15.56 \\
1.0 & 3.499 & 3.348 & 3.435 & 3.389 & 3.312 & 3.256 & 3.217 \\
1.5 & 4.467 & 4.353 & 4.451 & 4.410 & 4.306 & 4.233 & 4.158 \\
2.0 & 5.704 & 5.374 & 5.618 & 5.474 & 5.298 & 5.195 & 5.104 \\
2.5 & 6.312 & 6.184 & 6.339 & 6.270 & 6.118 & 6.011 & 5.896 \\
3.0 & 6.778 & 6.661 & 6.821 & 6.759 & 6.582 & 6.454 & 6.323 \\
3.5 & 7.465 & 7.150 & 7.468 & 7.299 & 7.040 & 6.881 & 6.720 \\
4.0 & 7.504 & 7.424 & 7.654 & 7.553 & 7.326 & 7.160 & 6.969 \\
4.5 & 7.596 & 7.577 & 7.772 & 7.705 & 7.473 & 7.301 & 7.102 \\
5.0 & 7.860 & 7.767 & 8.076 & 7.938 & 7.646 & 7.472 & 7.246 \\
5.5 & 7.946 & 7.902 & 8.212 & 8.066 & 7.781 & 7.616 & 7.408 \\
6.0 & 8.282 & 8.234 & 8.459 & 8.369 & 8.132 & 7.980 & 7.805 \\
6.5 & 8.611 & 8.666 & 8.862 & 8.797 & 8.561 & 8.406 & 8.197 \\
7.0 & 8.729 & 8.885 & 9.001 & 8.999 & 8.785 & 8.637 & 8.447 \\
7.5 & 8.992 & 9.094 & 9.261 & 9.222 & 8.989 & 8.821 & 8.620 \\
8.0 & 8.907 & 9.092 & 9.226 & 9.211 & 8.992 & 8.846 & 8.647 \\
8.5 & 9.007 & 9.096 & 9.387 & 9.237 & 8.986 & 8.797 & 8.598 \\
9.0 & 8.993 & 9.163 & 9.360 & 9.284 & 9.067 & 8.916 & 8.710 \\
9.5 & 8.830 & 9.052 & 9.176 & 9.164 & 8.966 & 8.843 & 8.677 \\
10.0 & 8.893 & 9.095 & 9.310 & 9.218 & 9.002 & 8.856 & 8.671 \\
10.5 & 8.805 & 9.048 & 9.283 & 9.181 & 8.950 & 8.809 & 8.641 \\
11.0 & 8.778 & 9.029 & 9.283 & 9.157 & 8.937 & 8.804 & 8.641 \\
11.5 & 8.596 & 8.903 & 9.081 & 9.014 & 8.820 & 8.702 & 8.541 \\
12.0 & 8.395 & 8.740 & 8.827 & 8.833 & 8.677 & 8.582 & 8.444 \\
12.5 & 8.445 & 8.756 & 8.928 & 8.858 & 8.680 & 8.571 & 8.419 \\
13.0 & 8.304 & 8.671 & 8.901 & 8.776 & 8.601 & 8.500 & 8.359 \\
13.5 & 8.133 & 8.477 & 8.717 & 8.586 & 8.407 & 8.310 & 8.174 \\
\hline & & & & & & & \\
\end{tabular}

Table D.2. Petrosion radii [arcsec] for the Kroupa model.

\begin{tabular}{|c|c|c|c|c|c|c|c|}
\hline redshift & petroR90 ${ }_{\mathrm{BOL}}$ & petroR90 ${ }_{V j}$ & $\overline{\text { petroR90 }}$ & petroR90 & petroR90 $r$ & petroR90 & $\overline{\mathrm{roR} 90_{z}}$ \\
\hline 9.5116 & 3.886 & 0 & 0 & 0 & 0 & 0 & 3.879 \\
\hline 5.6177 & 0.585 & 0.610 & 0.673 & 1.038 & 0.615 & 0.604 & 0.596 \\
\hline 4.0444 & 0.643 & 0.665 & 0.865 & 0.660 & 0.655 & 0.654 & 0.650 \\
\hline 3.1576 & 0.748 & 0.794 & 0.808 & 0.808 & 0.790 & 0.778 & 0.785 \\
\hline 2.5758 & 0.785 & 0.809 & 0.817 & 0.809 & 0.807 & 0.816 & 0.807 \\
\hline 2.1589 & 0.816 & 0.836 & 0.849 & 0.838 & 0.839 & 0.840 & 0.828 \\
\hline 1.8422 & 0.883 & 0.925 & 0.941 & 0.929 & 0.928 & 0.909 & 0.892 \\
\hline 1.5915 & 0.884 & 0.931 & 0.930 & 0.928 & 0.927 & 0.911 & 0.903 \\
\hline 1.3868 & 0.900 & 0.939 & 0.940 & 0.938 & 0.932 & 0.923 & 0.924 \\
\hline 1.2156 & 0.945 & 0.992 & 1.008 & 1.000 & 0.984 & 0.972 & 0.970 \\
\hline 1.0696 & 0.977 & 1.028 & 1.041 & 1.039 & 1.018 & 1.011 & 1.003 \\
\hline 0.9432 & 1.049 & 1.079 & 1.090 & 1.087 & 1.074 & 1.073 & 1.062 \\
\hline 0.8322 & 1.131 & 1.168 & 1.184 & 1.178 & 1.164 & 1.168 & 1.152 \\
\hline 0.7336 & 1.199 & 1.235 & 1.247 & 1.238 & 1.237 & 1.243 & 1.227 \\
\hline 0.6453 & 1.301 & 1.340 & 1.365 & 1.344 & 1.346 & 1.338 & 1.318 \\
\hline 0.5655 & 1.370 & 1.419 & 1.432 & 1.417 & 1.429 & 1.415 & 1.393 \\
\hline 0.4928 & 1.486 & 1.549 & 1.587 & 1.559 & 1.545 & 1.515 & 1.488 \\
\hline 0.4263 & 1.610 & 1.680 & 1.699 & 1.678 & 1.675 & 1.648 & 1.623 \\
\hline 0.3650 & 1.739 & 1.818 & 1.804 & 1.808 & 1.810 & 1.783 & 1.761 \\
\hline 0.3082 & 1.959 & 2.050 & 2.066 & 2.055 & 2.030 & 1.996 & 1.968 \\
\hline 0.2554 & 2.216 & 2.326 & 2.349 & 2.339 & 2.301 & 2.259 & 2.229 \\
\hline 0.2061 & 2.598 & 2.718 & 2.761 & 2.744 & 2.689 & 2.644 & 2.609 \\
\hline 0.1599 & 3.118 & 3.267 & 3.295 & 3.296 & 3.237 & 3.191 & 3.148 \\
\hline 0.1165 & 3.981 & 4.176 & 4.168 & 4.207 & 4.142 & 4.100 & 4.045 \\
\hline 0.0756 & 5.891 & 6.141 & 6.227 & 6.203 & 6.084 & 6.011 & 5.918 \\
\hline 0.0369 & 11.34 & 11.88 & 12.16 & 12.03 & 11.77 & 11.65 & 11.47 \\
\hline 0.0000 & 1678 & 1748 & 1798 & 1771 & 1734 & 1714 & 1686 \\
\hline
\end{tabular}


P. Westera et al.: Initial mass function effects on the colour evolution of disk galaxies, Online Material p 12

Table D.3. Concentration parameters for the Kroupa model.

\begin{tabular}{rrrrrrrrr}
\hline \hline Redshift & $C_{\mathrm{BOL}}$ & $C_{V j}$ & $C_{u}$ & $C_{g}$ & $C_{r}$ & $C_{i}$ & $C_{z}$ \\
\hline 9.5116 & - & - & - & - & - & - & - \\
5.6177 & - & - & - & - & - & - & - \\
4.0444 & - & - & - & - & - & - & - \\
3.1576 & - & - & - & - & - & - & - \\
2.5758 & - & - & - & - & - & - & - \\
2.1589 & - & - & - & - & - & - & - \\
1.8422 & - & - & - & - & - & - & - \\
1.5915 & 0.234 & 0.298 & 0.337 & 0.311 & 0.292 & 0.264 & 0.237 \\
1.3868 & 0.135 & 0.135 & 0.135 & 0.135 & 0.135 & 0.135 & 0.134 \\
1.2156 & 0.229 & 0.228 & 0.221 & 0.230 & 0.226 & 0.224 & 0.220 \\
1.0696 & 0.290 & 0.270 & 0.265 & 0.277 & 0.268 & 0.264 & 0.265 \\
0.9432 & 0.378 & 0.363 & 0.360 & 0.370 & 0.361 & 0.352 & 0.353 \\
0.8322 & 0.398 & 0.383 & 0.382 & 0.384 & 0.383 & 0.378 & 0.379 \\
0.7336 & 0.297 & 0.264 & 0.243 & 0.255 & 0.266 & 0.267 & 0.274 \\
0.6453 & 0.284 & 0.240 & 0.232 & 0.236 & 0.238 & 0.244 & 0.250 \\
0.5655 & 0.311 & 0.269 & 0.261 & 0.268 & 0.264 & 0.270 & 0.278 \\
0.4928 & 0.319 & 0.275 & 0.258 & 0.274 & 0.274 & 0.283 & 0.291 \\
0.4263 & 0.324 & 0.271 & 0.259 & 0.271 & 0.273 & 0.282 & 0.291 \\
0.3650 & 0.353 & 0.303 & 0.322 & 0.313 & 0.303 & 0.306 & 0.311 \\
0.3082 & 0.371 & 0.314 & 0.329 & 0.320 & 0.315 & 0.319 & 0.323 \\
0.2554 & 0.387 & 0.333 & 0.338 & 0.334 & 0.334 & 0.339 & 0.344 \\
0.2061 & 0.410 & 0.343 & 0.362 & 0.346 & 0.343 & 0.347 & 0.352 \\
0.1599 & 0.441 & 0.373 & 0.399 & 0.378 & 0.373 & 0.375 & 0.382 \\
0.1165 & 0.456 & 0.397 & 0.434 & 0.405 & 0.394 & 0.393 & 0.401 \\
0.0756 & 0.492 & 0.417 & 0.463 & 0.425 & 0.415 & 0.416 & 0.427 \\
0.0369 & 0.517 & 0.438 & 0.470 & 0.443 & 0.438 & 0.441 & 0.455 \\
0.0000 & 0.556 & 0.479 & 0.512 & 0.484 & 0.478 & 0.481 & 0.497 \\
\hline & & & & & & &
\end{tabular}

